\title{
Inhibition of relaxin autocrine signaling confers therapeutic vulnerability in ovarian cancer
}

\author{
Helen E. Burston, ${ }^{1}$ Oliver A. Kent, ${ }^{1}$ Laudine Communal, ${ }^{2,3}$ Molly L. Udaskin, ${ }^{1}$ Ren X. Sun, ${ }^{1}$ Kevin R. Brown, ${ }^{4}$ Euihye Jung, ${ }^{5}$ \\ Kyle E. Francis, ${ }^{1}$ Jose La Rose, ${ }^{1}$ Joshua Lowitz, ${ }^{6}$ Ronny Drapkin, ${ }^{5}$ Anne-Marie Mes-Masson, ${ }^{2,3,7}$ and Robert Rottapel ${ }^{1,8,9}$

\begin{abstract}
'Princess Margaret Cancer Centre, University Health Network (UHN), Toronto, Ontario, Canada. ²entre de Recherche du Centre Hospitalier de l'Université de Montréal (CRCHUM), Montreal, Quebec, Canada. ${ }^{3}$ Institut du Cancer de Montréal, Montréal, Quebec, Canada. “Banting and Best Department of Medical Research, Donnelly Centre for Cellular and Biomolecular Research, University of Toronto, Toronto, Ontario, Canada. ${ }^{5}$ Penn Ovarian Cancer Research Center, University of Pennsylvania, Philadelphia, Pennsylvania, USA. ${ }^{6}$ Antibody Solutions, Santa Clara, California, USA. 'Département de Médecine, Université de Montréal, Montreal, Quebec, Canada. ${ }^{8}$ Department of Medical Biophysics, Department of Immunology, University of Toronto, Toronto, Ontario, Canada. ${ }^{9}$ Division of Rheumatology, St. Michael's Hospital, Toronto, Ontario, Canada.
\end{abstract}

\begin{abstract}
Ovarian cancer (OC) is the most deadly gynecological malignancy, with unmet clinical need for new therapeutic approaches. The relaxin peptide is a pleiotropic hormone with reproductive functions in the ovary. Relaxin induces cell growth in several types of cancer, but the role of relaxin in OC is poorly understood. Here, using cell lines and xenograft models, we demonstrate that relaxin and its associated CPCR RXFP1 form an autocrine signaling loop essential for OC in vivo tumorigenesis, cell proliferation, and viability. We determined that relaxin signaling activates expression of prooncogenic pathways, including RHO, MAPK, Wnt, and Notch. We found that relaxin is detectable in patient-derived OC tumors, ascites, and serum. Further, inflammatory cytokines IL- 6 and TNF- $\alpha$ activated transcription of relaxin via recruitment of STAT3 and NF- $\kappa$ B to the proximal promoter, initiating an autocrine feedback loop that potentiated expression. Inhibition of RXFP1 or relaxin increased cisplatin sensitivity of $\mathrm{OC}$ cell lines and abrogated in vivo tumor formation. Finally, we demonstrate that a relaxin-neutralizing antibody reduced $\mathrm{OC}$ cell viability and sensitized cells to cisplatin. Collectively, these data identify the relaxin/RXFP1 autocrine loop as a therapeutic vulnerability in OC.
\end{abstract}

\section{Introduction}

Ovarian cancer (OC) has the highest mortality rate of all gynecological malignancies. High-grade serous ovarian carcinoma (HGSOC) is the most common and aggressive subtype, with a dismal 5-year survival rate of $30 \%$ (1). Poor survival has been linked to early asymptomatic metastatic progression accompanied by dissemination of cancer cells throughout the peritoneal cavity (2). The standard treatment for HGSOC is cytoreductive surgery followed by platinum-based chemotherapy. Although most women initially respond to chemotherapy, the majority of patients will suffer disease recurrence following treatment and $70 \%$ will die of recurrent disease within 5 years of initial diagnosis (3). There is an urgent unmet need to identify new targets that can be drugged therapeutically. Targeted therapies may demonstrate greater selectivity, lower toxicity, and improved outcome over conventional chemotherapy (4).

GPCRs and their ligands underlie several aspects of cancer initiation and progression, including aberrant cell proliferation, metastasis, adhesion, and angiogenesis (5). GPCR-ligand interactions are highly druggable, which makes them attractive for

Conflict of interest: Conflict of interest statement: The authors have declared that no conflict of interest exists.

Copyright: @ 2021, American Society for Clinical Investigation.

Submitted: July 24, 2020; Accepted: February 3, 2021; Published: April 1, 2021.

Reference information: J Clin Invest. 2021;131(7):e142677.

https://doi.org/10.1172/JCl142677. cancer drug-discovery research (6). The GPCR encoding the relaxin receptor (RXFP1) and its ligand relaxin regulate pleiotropic functions across a variety of tissues (7). Relaxin family peptides are part of the insulin superfamily and include both relaxin and insulin-like (INSL) peptides. Relaxin plays a central role in reproduction and mediates follicle growth, endometrial differentiation, and uterine angiogenesis prior to implantation. The ovaries and prostate are the 2 major sources of relaxin in humans. Relaxin-2 (RLN2) and the highly similar $R L N 1$ are coexpressed at low levels in multiple tissues, including the decidua, placenta, endometrium, prostate, and myocardium, and act both as autocrine and paracrine hormones (8). Relaxin peptides are processed from a propeptide form (pro-RLN) to produce mature peptides containing an A-chain linked to a B-chain by 2 disulphide bonds (7). Upon activation, RXFP1 couples to the small G proteins $G \alpha_{s}$ and $G \alpha_{i 3}$ to initiate production of cAMP as well as activation of PI3K and the RAF/MEK/ERK (MAPK) signaling pathways $(7,9)$. There is increasing evidence that relaxin may play a central role in multiple cancers, particularly cancers of reproductive origin (10).

Here, we identified relaxin/RXFP1 as an essential autocrine loop in a subset of OC cell lines. We explored the role of the relaxin/RXFP1 signaling pathway in the initiation and progression of ovarian tumorigenesis, its activation by inflammatory mediators, and contribution to platinum resistance and developed an antibody-based reagent targeting RLN2 with therapeutic potential. 


\section{Results}

The GPCR RXFP1 is essential for survival in a subset of OC cells. To identify GPCRs that support the survival of OC cells, a genomewide screening in 33 epithelial OC cell lines was performed: 28 HGSOC, 1 clear cell, and 4 of unknown histotype based on the suitability scores described by Medrano et al. (11), Marcotte et al. (12), Domcke et al. (13), and Beaufort et al. (14) (Supplemental Figure 1A; supplemental material available online with this article; https://doi.org/10.1172/JCI142677DS1). Twenty-two of the cell lines were derived from individual patients, and the remaining were isogenic pairs derived from 1 of these 22 lines (11). Each receptor was ranked based on the frequency of essentiality across cell lines (zGARP-associated $P$ value, $P<0.05$ ). The screen contained shRNAs targeting 376 GPCRs and revealed the relaxin receptor $R X F P 1$ as the most frequently essential GPCR, with 19 lines demonstrating dependency (Figure 1A and Supplemental Figure 1A). There are 4 relaxin-related receptor genes in the human genome. RXFP1 and RXFP2 share $60 \%$ amino acid sequence homology, while RXFP3 and RXFP4 are considerably more divergent (15). RXFP2 was essential in 6 cell lines, 3 of which were also dependent on RXFP1 (Supplemental Figure 1A).

$R X F P 1$ expression was assessed in OC organoids established from 2 patients with HGSOC (OC-organoid 1 and OC-organoid 2). Both stained positively for PAX8, which is expressed in $80 \%$ to $96 \%$ of HGSOCs (16). OC-organoid 1 showed a loss of TP53 expression, while OC-organoid 2 demonstrated strong positive staining, indicative of a stabilizing TP53 mutation (Figure 1B). Both organoids stained positively for RXFP1, confirming expression in this HGSOC model system. RXFP1 expression was detected in squamous epithelial cells (Supplemental Figure 1C), consistent with the reported staining for the Human Protein Atlas (HPA) approved antibody (17).

To validate essentiality of RXFP1 in secondary screens, 8 OC cell lines were selected, demonstrating a range of RFXP1 expression and dependency (Supplemental Figure 1, A and B). The cell line panel included OVCAR8, SKOV3, PEO4, PEO6, OAW42, PEA1, PEA2, and OVCAR5. Each was infected with 2 independent shRNAs (sh1-RXFP1 or sh2-RXFP1) targeting $R X F P 1$, a nontargeting control shRNA (shGFP), or shRNA targeting the essential gene PSMD1 (sh-PSMD1). Knockdown of RXFP1 potently impaired proliferation of OVCAR8, SKOV3, PEO4, and PEO6, whereas OAW42 and PEA2 demonstrated intermediate dependency (Figure 1C and Supplemental Figure 1, D and E). PEA1 and OVCAR5, which do not express detectable levels of $R X F P 1$, were resistant to knockdown. Interestingly, OVCAR8 carries missense mutations in both RXFP2 (L737F) and RXFP3 (T76P) (Cancer Cell Line Encyclopedia; broadinstitute.org/ccle). RXFP2-L737F is contained within the N-terminal cytosolic region, which forms the docking site for $\mathrm{G \alpha}_{\mathrm{i} 3}$, while RXFP3-T76P is contained within the receptor ligand binding/ extracellular domain. As we were unable to detect expression of RXFP2, the effects of RXFP2 knockdown could not be evaluated. Both OVCAR8 and SKOV3 express higher levels of $\mathrm{Ga}_{\mathrm{i} 3}$ relative to other cell lines (BioGPS Cell Line Gene Expression Profiles), suggesting that RXFP1 dependency could arise from expression and function of RXFP1 and related receptors as well as downstream signaling components.
OVCAR8 and SKOV3 depleted of RXFP1 demonstrated a rounded morphology suggestive of apoptosis activation (Figure 1D). Consistent with this phenotype, increased PARP and caspase-3 cleavage decreased expression of the antiapoptotic protein BCL2 and increased propidium iodide (PI) annexin V staining, confirming apoptosis was observed (Figure 1E and Supplemental Figure 1F). OVCAR5 showed no change in PI annexin V staining following RXFP1 knockdown (Supplemental Figure $1 \mathrm{~F}$ ). RXFP1 is therefore required for survival in a subset of OC cell lines.

$R X F P 1$ is essential for tumorigenesis of OC cells. The contribution of RXFP1 to tumorigenic phenotypes in OC cells was next examined. Knockdown of RXFP1 decreased anchorage-independent growth of OVCAR8 and SKOV3, but had no effect on OVCAR5 (Figure $1 \mathrm{~F}$ and Supplemental Figure 1G). RXFP1 knockdown in OVCAR8 impaired tumor formation when injected into the mammary fat pad (MFP) of NOD/SCID/IL2R $\gamma^{-/-}$(NSG) mice compared with shGFP control (Supplemental Figure 1, I-L).

To determine whether RXFP1 was essential for sustained tumor growth, OVCAR8 were engineered to express a tetracycline-inducible (TET-inducible) shRNA targeting RXFP1 (TETsh1-RXFP1) or control shGFP (TET-shGFP). Induction of shRNA expression with doxycycline (Dox) resulted in knockdown of RXFP1 mRNA with a concomitant decrease in cell viability compared with OVCAR8 grown in the absence of Dox (Figure $1 G$ and Supplemental Figure 1M). In the absence of Dox, OVCAR8derived tumors containing TET-shGFP or TET-sh1-RXFP1 demonstrated robust growth (Figure 1, H-J). In contrast, mice given Dox from onset (Dox D0) demonstrated a significant reduction in tumor growth relative to control groups. Mice given Dox 21 days after injection ( 2 weeks following the appearance of measurable tumors) demonstrated a reduction in tumor growth, with final tumor volumes approximately $50 \%$ smaller than tumors in control groups (Figure 1, H-J). Thus, RXFP1 contributed to both OC tumor initiation and progression in xenograft models.

Relaxin activates proliferative signaling pathways via $R X F P 1$. Given the deleterious effect of RXFP1 knockdown, the effect of relaxin on OC cells was examined. Recombinant human relaxin (rhRLN2) induced increased viability in OVCAR8 and SKOV3 (Figure 2A). Relaxin stimulation increased BrdU incorporation and activated MAPK and AKT signaling in OVCAR8, which was blocked in cells with RXFP1 knockdown, corroborating that relaxin-induced signaling is dependent upon $R X F P 1$ expression (Figure 2, B and C, and Supplemental Figure 2A). Relaxin stimulation promoted 5 -fold increased cAMP production in OVCAR8 (Supplemental Figure 2B).

To discover the transcriptional program induced by relaxin, RNA-Seq was performed in OVCAR8 treated with rhRLN2. In total, 766 mRNAs were upregulated and 73 mRNAs downregulated in response to rhRLN2 treatment. Among the upregulated mRNAs were known relaxin target genes, including VEGF and MMPs (18, 19). Enrichment analysis revealed gene signatures including RHO GTPase signaling, extracellular matrix regulation, cell adhesion, actin cytoskeleton, and signaling via MAPK, WNT, and NOTCH (Figure 2D). Relaxin-induced target genes involved in tissue remodelling and angiogenesis (VEGFA, MMP9, MMP23), Notch signaling (NOTCH1, NOTCH3), transcription (FOXL2, SOX12, $A R I D 5 A)$, and WNT signaling (DVL1, BCL9, LRP5) were validated 
A

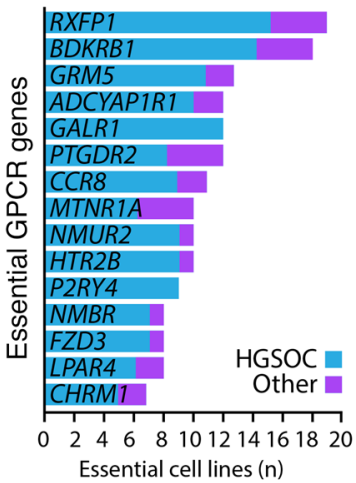

B

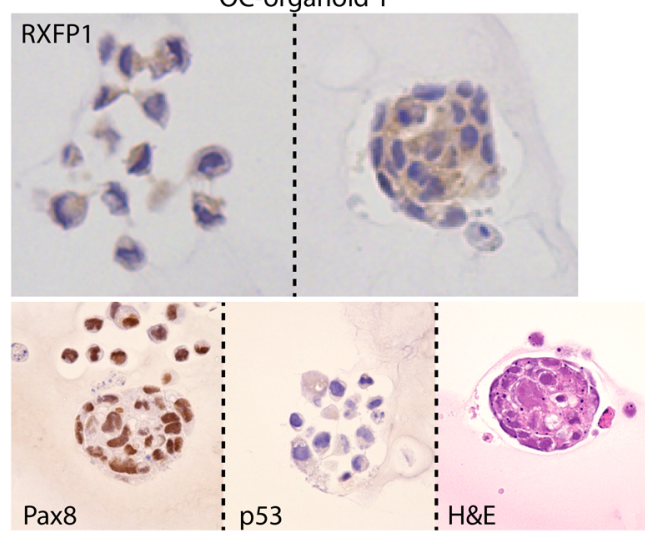

C

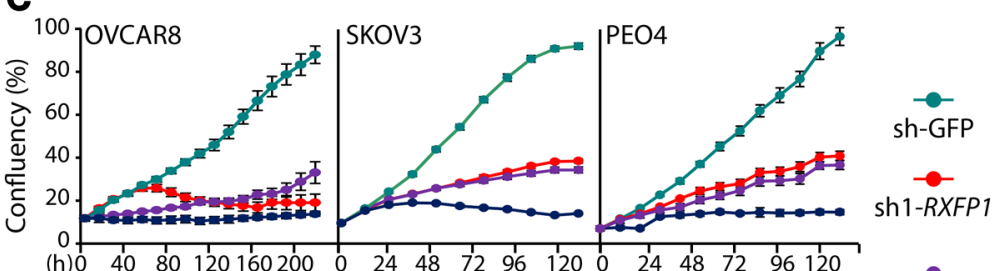

D
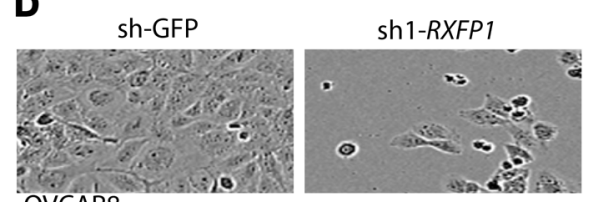

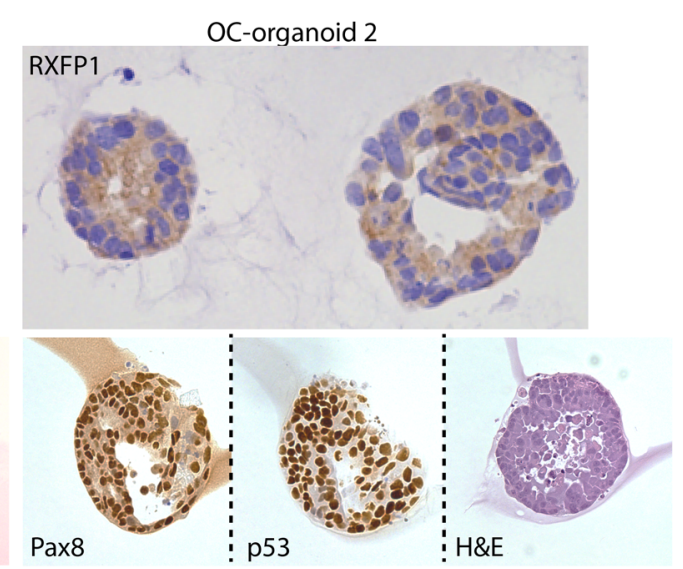

OVCAR8
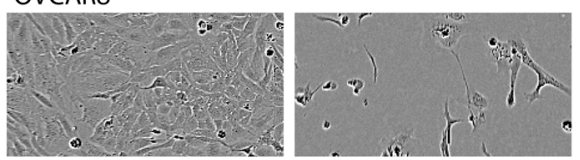

SKOV3

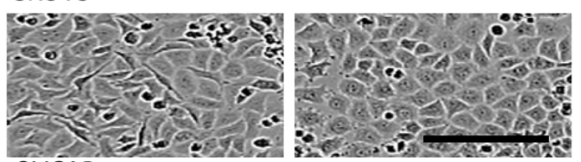

OVCAR5
E

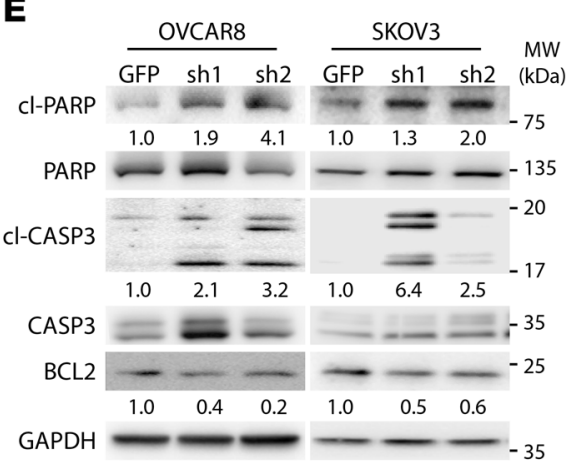

H

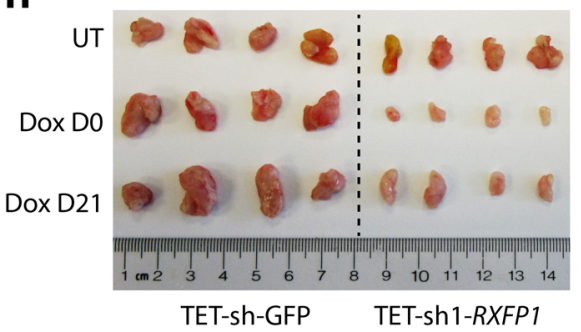

$\mathbf{F}$

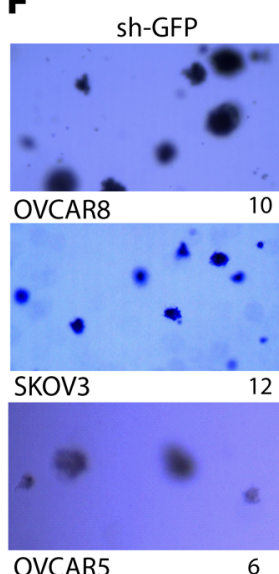

I

- TET-sh-GFP

$\rightarrow$ TET-Sh1-RXFP1

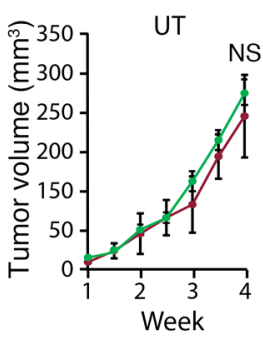

Sh1-RXFP1
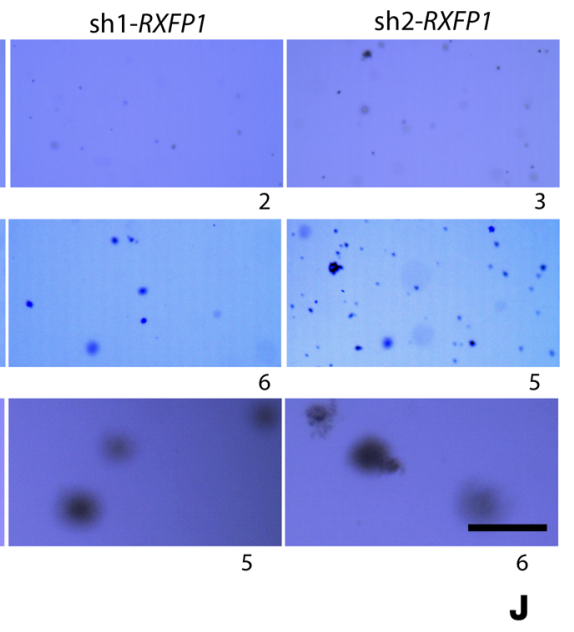

$\mathbf{J}$

G UT

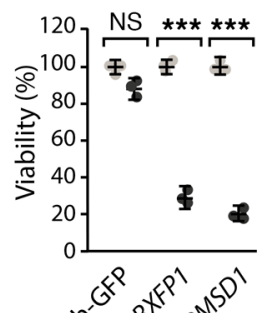
在

TET-sh-GFP

TET-sh1-RXFP1
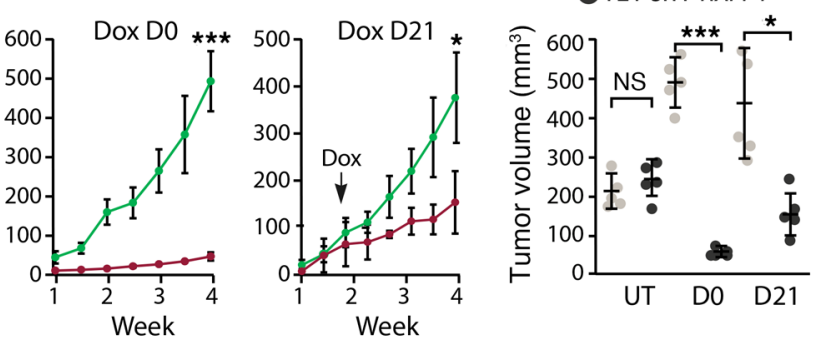
Figure 1. RXFP1 is an essential GPCR in OC cell lines. (A) GPCRs identified by shRNA screening. Genes are arranged by number of dependent cell lines based on significance of the normalized zGARP score $(<0.05)$. Other represents clear cell or unknown origin. (B) RXFP1, Pax8, and TP53 staining in OC organoids. Original magnification, $\times 20 . n=2$. (C) Growth of cell lines constitutively expressing shRNA control (shGFP), shRNAs targeting RXFP1 (sh1-RXFP1 or sh2-RXFP1), or targeting PSMD1 (sh-PSMD1). Data points represent mean \pm SEM. $n=3$. (D) Images of OVCAR8, SKOV3, and OVCAR5 at 72 hours after infection with the indicated constitutively expressed shRNAs. Scale bar: $5 \mu \mathrm{m}$. (E) Analysis of apoptosis in OVCAR8 and SKOV3 constitutively expressing shGFP (GFP) or shRNA targeting RXFP1 (sh1 or sh2) 72 hours after infection. (F) Soft agar growth of cells constitutively expressing shGFP or shRNA targeting RXFP1 (sh1 or sh2). Average colony counts are indicated; also see Supplemental Figure 1G. Scale bar: $100 \mu \mathrm{m}$. $n=3$. (G) Viability of OVCAR8 expressing Dox-inducible TET-shGFP,

TET-sh1-RXFP1, or TET-sh-PSMD1 in the absence or presence of Dox (+Dox, $1 \mu \mathrm{g} / \mathrm{mL}$ ) compared with untreated cells (UT). Data are represented as mean \pm SEM. $n=3 .{ }^{* *} P<0.001$, Student's $t$ test. $($ H) OVCAR8-derived xenografts expressing Dox-inducible control TET-shGFP or TET-sh1-RXFP1. Dox was initiated on day of cell injection (DO) or 21 days after injection (D21). (I) Analysis of OVCAR8 xenograft tumor measurement. Arrow indicates when Dox treatment was initiated (21 days after injection). Data are represented as mean \pm SEM. $n=4 .{ }^{*} P<0.05 ;{ }^{* *} P<0.00001$, Student's $t$ test. (J) Final mean volume ( \pm SEM) of tumors described in $\mathbf{I} .{ }^{*} P<0.05$; ${ }^{* *} P<0.001$, Student's $t$ test.

(Figure 2E and Supplemental Figure 2C). CRISPR dropout screens revealed that OVCAR8 and SKOV3 shared common genetic dependencies on relaxin-induced genes (Supplemental Figure 2D and refs. 20-22). Amplification of relaxin-regulated genes was found in a large percentage (5\%-18\% per gene) of HGSOC tumors in The Cancer Genome Atlas (TCGA) data set (Supplemental Figure $2 \mathrm{E}$ and refs. 23-25). Relaxin signaling therefore results in the activation of multiple pathways supporting tumorigenesis.

Relaxin autocrine signaling promotes survival in OC cells. The human genome contains 2 relaxin genes, RLN1 and RLN2, expressed from $R L N 1$ and $R L N 2$, respectively, which share $84 \%$ protein sequence identity; both activate RXFP1 (26). For clarity, we refer to the human relaxin peptides/protein collectively as relaxin and genes as RLN. There is reported crosstalk between relaxin-related ligands and receptors (Figure 3A). In addition to RXFP1, relaxin can also interact with RXFP2, although with weaker affinity (27). INSL3 is specific for RXFP2. The highly divergent $R L N 3$, the cognate ligand for RXFP3, is also capable of RXFP1 activation, but is expressed predominantly in the brain (28).

RNA-Seq analysis revealed that RLN1 and RLN2 were coexpressed in OC cell lines at low but detectable levels, with $R L N 2$ generally exhibiting higher expression relative to RLN1 (Supplemental Figure 3A). RLN3 expression was undetectable (fragments per kilobase of transcript per million $[\mathrm{FPKM}]=0$ ) in the majority of lines. OVCAR8 and SKOV3 expressed predominantly $R L N 1$, while PEA2 primarily expressed $R L N 2$ (Figure 3B). Expression of $R L N$ in OC cells correlated with dependency on RXFP1. To determine whether OC cells exhibited relaxin dependency, RLN1 and RLN2 were knocked down using 2 independent shRNAs (sh1-RLN and sh2-RLN) that target sequences common to both $R L N 1$ and RLN2 (Supplemental Figure 3B) and confirmed to knock down RLN1 in OVCAR8 and SKOV3 and RLN2 in PEA2 (Supplemental Figure 3, C-E). OVCAR5 demonstrated decreased expression of both RLN1 and RLN2 in cells expressing sh1-RLN and sh2-RLN (Supplemental Figure 3, C-D). Relaxin was detectable in supernatants collected from OVCAR8, SKOV3, PEA2, and OVCAR5, but reduced in cells expressing sh1-RLN and sh2-RLN, confirming the specificity of the shRNA (Figure 3C and Supplemental Figure 3, E and F). Knockdown of RLN increased cleavage of PARP and CASP3 and decreased levels of BCL2 in OVCAR8 and SKOV3 compared with shGFP control (Figure 3D).

Knockdown of RLN phenocopied knockdown of RXFP1 in the panel of cell lines tested (Figure 3E), with OVCAR8 and SKOV3 being the most dependent and OVCAR5 the least dependent. Consistent with dependency of the ligand-receptor pair, RLN knockdown abrogated colony formation in OVCAR8 and SKOV3, but not in OVCAR5 (Figure 3F and Supplemental Figure 3G)

OVCAR8 expressing sh1-RLN had a delayed onset of tumorigenesis, with tumors being undetectable until 3 weeks after injection (Figure 3, G and H). In contrast, OVCAR8 expressing shGFP formed measurable tumors in NSG mice 1.5 weeks after injection. Tumors established from OVCAR8-shGFP reached a final volume of $550 \mathrm{~mm}^{3}$, whereas tumors from OVCAR8-sh1-RLN were $45 \%$ smaller and reached an average volume of $300 \mathrm{~mm}^{3}$ (Figure 3, $\mathrm{G}$ and $\mathrm{H}$, and Supplemental Figure $3 \mathrm{H})$. Positive CD31 staining, a marker of microvasculature $(29,30)$, was observed in shGFP control tumors and significantly decreased in tumors expressing shRLN (Figure 3, I and J, and Supplemental Figure 3I). Therefore, relaxin is required for optimal growth in a subset of OC cells both in vitro and in vivo and supports angiogenesis.

Relaxin expression is induced by inflammatory cytokines in OC-derived ascites. HGSOCs originate in the secretory cells of the fallopian tube (FT) (31). Following oncogenic transformation, these cells disseminate into the peritoneal cavity, where they undergo metastatic colonization (2). The FT epithelium is an extraovarian source of relaxin and expresses both $R L N 1$ and $R L N 2$ $(32,33)$. Expression of relaxin was observed in FT secretory cells, OE6/E7 oviductal cells, and 9 of 12 OC cell lines, but was undetectable in all 5 cell lines derived from normal ovarian epithelium (Figure 4A). Consistent with relaxin/RXFP1 dependency in cancer cell survival and the nontumorgenic potential of FT cells, shRNA knockdown of either RXFP1 or RLN had no effects on cell viability in FT194 (Supplemental Figure 4A).

Relaxin expression was evaluated in primary HGSOC tumors and normal FT samples derived from the Canadian Ovarian Experimental Unified Resource (COUER) cohort. The analysis of relaxin expression included 73 primary HGSOC tumors and 9 normal FT samples. Analysis revealed relaxin expression in FT epithelium and HGSOC tumors, with a range of expression observed in tumors (Figure 4B and Supplemental Figure 4, B and C). Quantification revealed a larger but nonsignificant variation in relaxin expression in tumors compared with FT cells (Supplemental Figure 4D). Sera derived from a patient cohort consisting of HGSOC samples $(n=38)$, clear cell carcinomas $(n=3)$, other OCs $(n=7)$, and control serum from healthy donors $(n=14)$ were also evaluated (Supplemental Figure 4E). Relaxin levels were significantly higher in blood serum from the 48 OC patients relative to that from 14 normal controls ( $P=0.01$; Figure $4 \mathrm{C})$.

Peritoneal ascites develops in two-thirds of advanced stage OCs, contributing extensively to morbidity (34). Malignant ascites is a reservoir of soluble factors that provide a proinflammatory and 
A

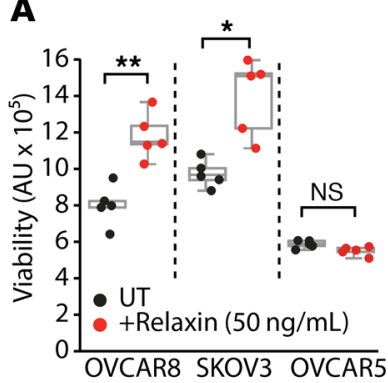

B

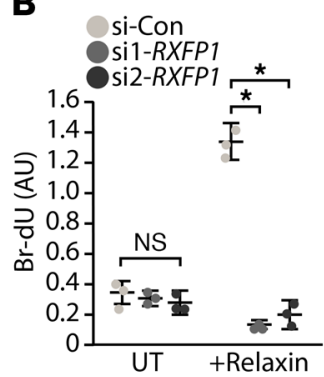

C

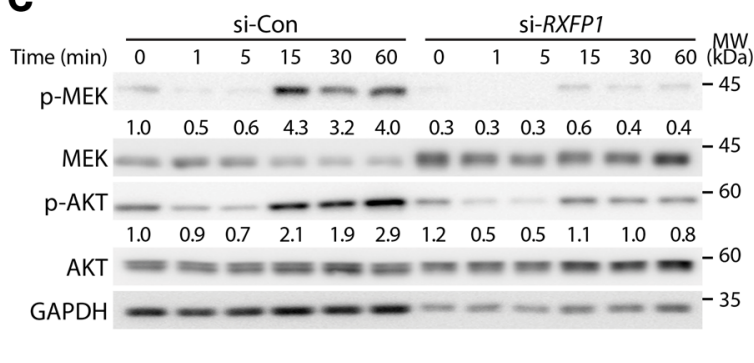

D

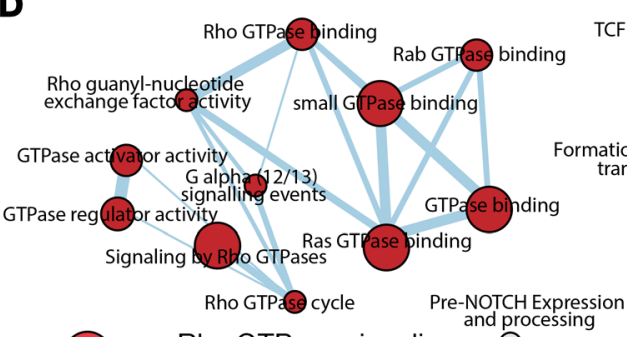

Kina binding Rho GTPase signaling MAPOKinase binding MAPK signaling (a) Nater Signalng by NOTCH NOTCH signaling
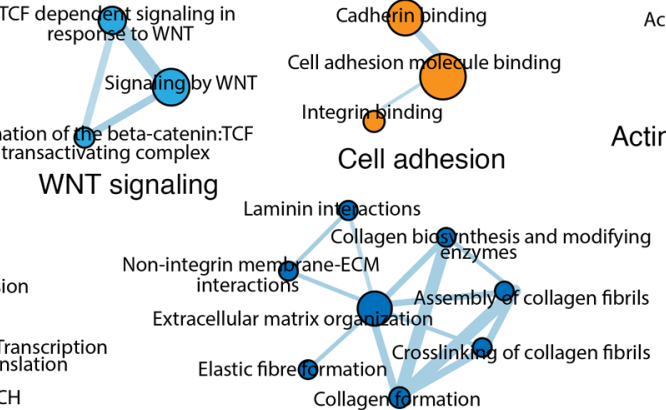

Collagenformation

Extracellular matrix

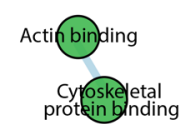

Actin cytoskeleton

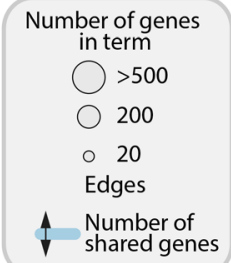

E

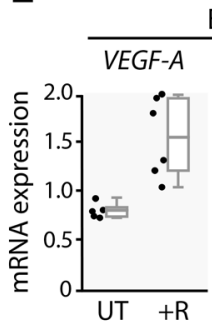

Extracellular matrix
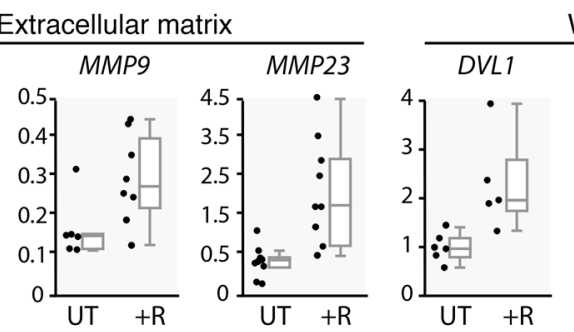

WNT signaling
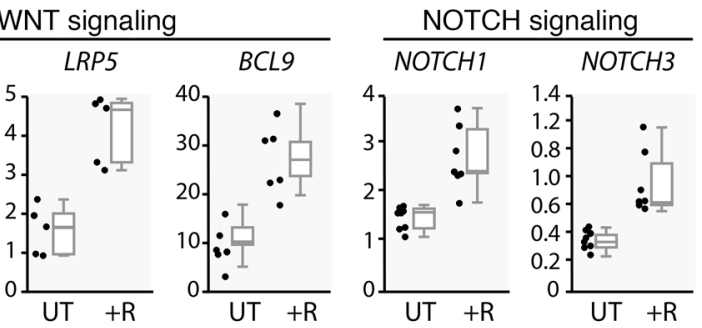

Figure 2. Relaxin initiates signaling pathways and gene activation. (A) Cell viability in the absence (untreated [UT]) or presence of recombinant human RLN2 (+Relaxin, $50 \mathrm{ng} / \mathrm{mL}$ ) for 24 hours. Results represent absorbance unit (AU) measurements $(n=5)$. Data are represented as mean \pm SEM. ${ }^{*} P<0.05$; ${ }^{* *} P<0.01$, Student's $t$ test. (B) BrdU incorporation in OVCAR8 in the absence (untreated) or presence of recombinant RLN2 (+Relaxin, 50 $\mathrm{ng} / \mathrm{mL}$ ) following transfection with control siRNA (siCON) or 2 different siRNAs targeting RXFP1 (si1-RXFP1 and si2-RXFP1). Results represent absorbance unit measurements. $n=3$. Data are represented as mean \pm SEM. (C) Analysis of p-MEK and p-AKT in OVCAR8 treated with human RLN2 (50 ng/ $\mathrm{mL}$ ) following transfection with siRNA control (siCON) or si-RXFP1. (D) Significantly enriched pathways identified by RNA-Seq (FDR Q value $<0.01$ ) in RLN2-treated OVCAR8. Nodes represent enriched pathways and edges the number of genes overlapping between 2 pathways. Enrichment analysis was carried out using g-profiler and visualized using Cytoscape. (E) QPCR analysis of the indicated mRNA transcripts in untreated cells or cells treated with $\mathrm{RLN2}$ (+R, $50 \mathrm{ng} / \mathrm{mL}$ for 8 hours). Data points represent individual wells/replicates. Box plots indicate the interquartile range (IQR) of the data, and the central line shows the median. $n \geq 5$.

tumor-promoting microenvironment (35). Relaxin was detected in 10 of 11 patient-derived ascites supernatants by ELISA (Figure $4 \mathrm{D}$ ). We conjectured that ascites may promote OC progression, in part by inducing relaxin expression. Consistent with this, FT194 had increased levels of relaxin following growth in 10\% ascites supernatant (Figure $4 \mathrm{E}$ ), suggesting that a soluble factor or factors present in ascites may contribute to elevated relaxin.

IL-6 is among the most abundant cytokine in ascites, and high levels predict worse progression-free survival in patients with advanced OC (36-38). High IL-6 and TNF- $\alpha$ have been identified in a subgroup of patients, which suggests an interaction between ascites IL- 6 and TNF- $\alpha$ driving tumor progression and resistance to chemotherapy (36). High levels of IL-6 were confirmed in patient-derived ascites (Supplemental Figure 4F). Relaxin can stimulate macrophages to produce IL-6, suggesting that relaxin signaling in tumor cells could be initiated and sustained in the tumor microenvironment through interaction with immune cell effectors $(39,40)$. Relaxin-stimulated OVCAR8 increased expression of IL-6 mRNA in a dose-dependent manner, but no effect on TNF mRNA was observed (Figure $4 \mathrm{~F}$ ).

We hypothesized that relaxin/IL-6 constituted a feedback loop; therefore, the effect of IL- 6 on relaxin expression was examined. Relaxin levels increased in OVCAR8 following treatment with IL-6 and reached maximal expression in OVCAR8 and SKOV3 16 hours after treatment (Figure $4, \mathrm{G}$ and H). TNF- $\alpha$ treatment also increased relaxin expression in OVCAR8 (Figure $4 \mathrm{G}$ ). Elevated RLN1 mRNA was observed in OVCAR8 following IL- 6 or TNF- $\alpha$ treatment, suggesting a transcriptional mecha- 
A

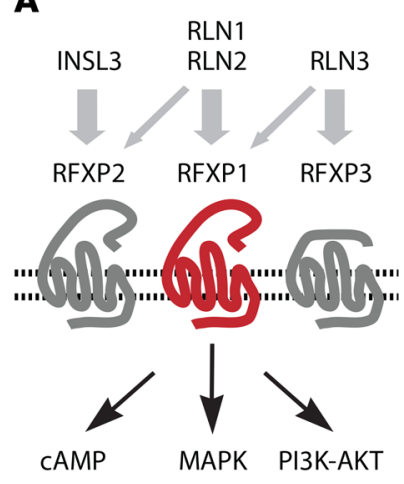

B

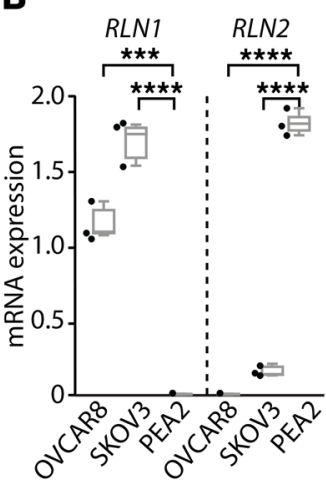

C

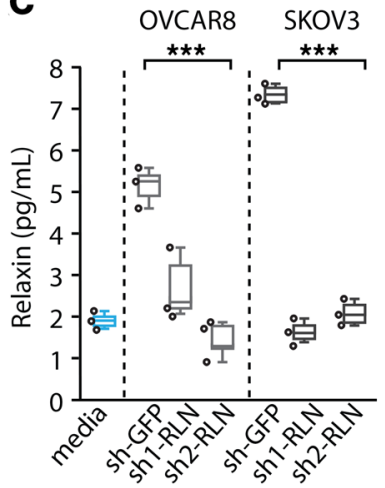

D

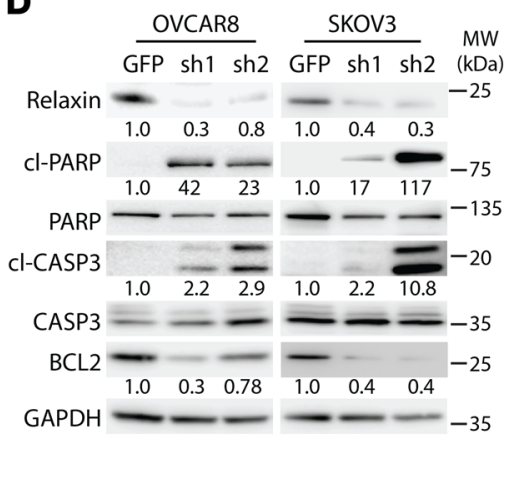

E
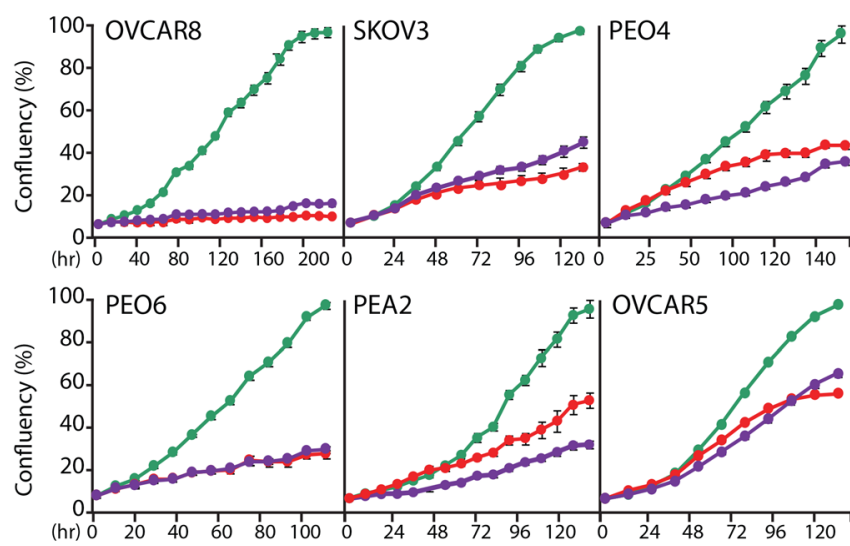

sh-GFP sh1-RLN sh2-RLN

G

sh-GFP

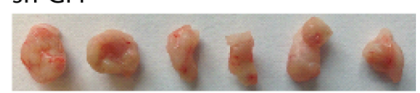

sh1-RLN

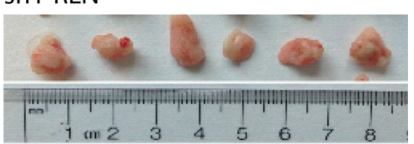

H

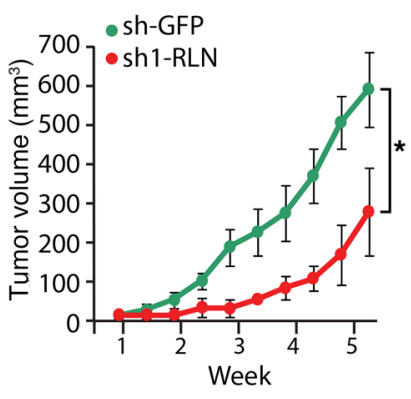

$\mathbf{F}$

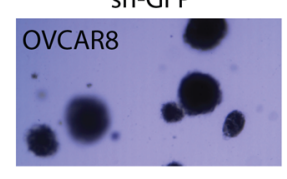

20

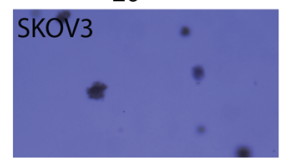

12

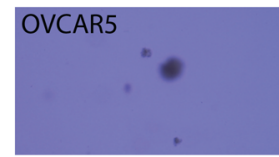

5

I

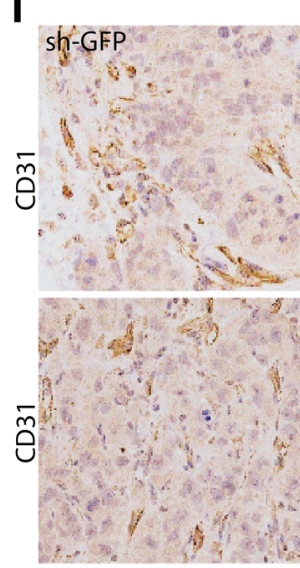

sh1-RLN

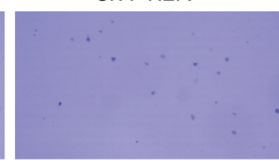

1

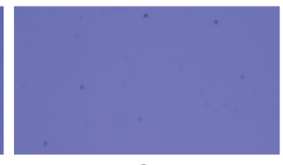

2

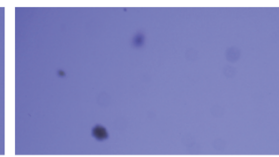

6

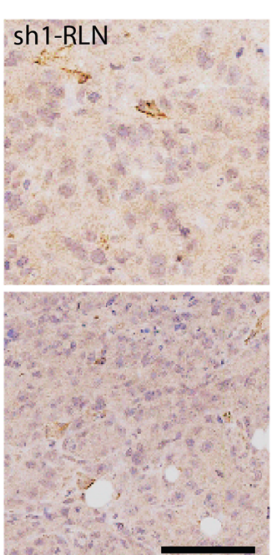

sh2-RLN

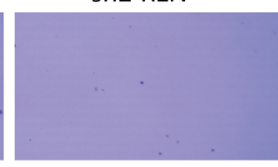

2

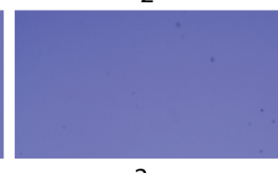

2

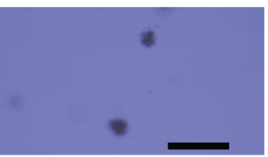

5

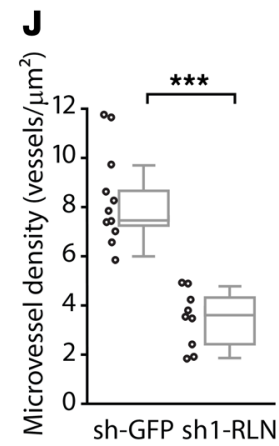

Figure 3. Expression of relaxin in OC cell lines is essential for survival. (A) Schematic of relaxin signaling via RXFP receptors (RXFP1, RXFP2, RXFP3) and ligands (RLN1/RLN2/RLN3/INSL3) summarizing ligand specificity, relative potency, and reported cross reactivity. (B) mRNA levels of RLN1 and RLN2 in OVCAR8, SKOV3, and PEA2. For panels B and C, box plots indicate the IQR of the data, and the central line shows the median. ${ }^{* * *} P<0.001 ;{ }^{* * *} P<$ 0.0001 , Student's $t$ test. (C) Relaxin levels in media derived from OVCAR8 and SKOV3 constitutively expressing shGFP or shRNA targeting RLN (sh1- or sh2-RLN) 120 hours following selection. $n=3 .{ }^{* *} P<0.001$, Student's $t$ test. (D) Analysis of prorelaxin- and apoptosis-related factors in OVCAR8 and SKOV3 constitutively expressing shGFP or shRNA targeting RLN (sh1- or sh2-) 48 hours following selection. cl-PARP, cleaved PARA; cl-CASP3, cleaved caspase-3. (E) Growth of OC cell lines constitutively expressing shGFP or shRNA targeting RLN. Data are represented as mean \pm SEM. $n=3$. (F) Soft agar growth of cell lines expressing shGFP or shRNA targeting RLN. Average colony counts are indicated; also see Supplemental Figure 3G. Scale bar: $100 \mu \mathrm{m}$. $n=3$. (G) Tumors derived from OVCAR8 expressing shGFP or sh1-RLN. (H) Growth curves of tumors described in G. ${ }^{*} P<0.02$, Student's $t$ test. (I) Representative images of CD31 IHC in shGFP control and sh1-RLN-expressing tumors. Scale bar: $10 \mu \mathrm{m}$. (J) Quantification of MVD (CD31-positive clusters per unit area) in CD31-enriched regions within tumors expressing shCFP ( $n=11$ regions) or sh1-RLN ( $n=9$ regions). Box plots indicate the IQR of the data, and the central line shows the median. ${ }^{* * *} P<0.0001$, Student's $t$ test. 
A

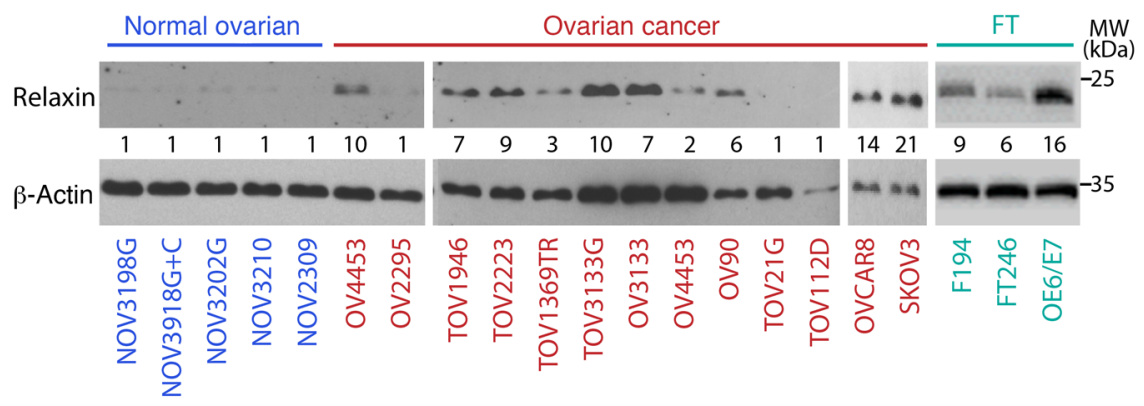

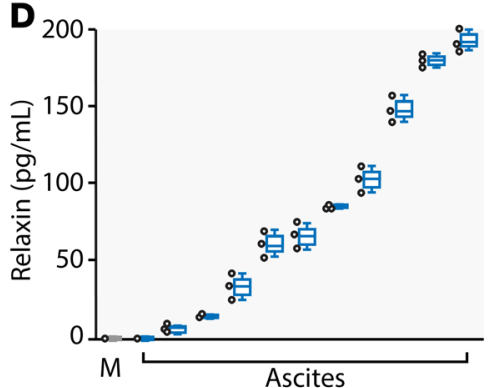

H

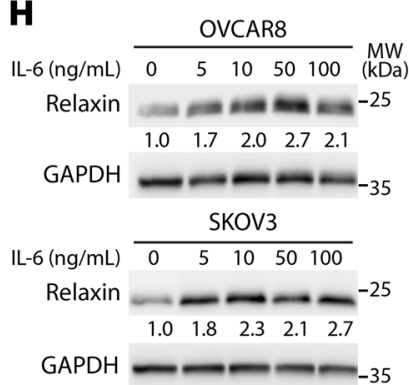

GAPDH $=-35$
B

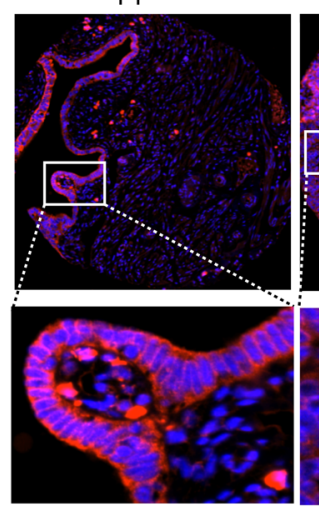

E

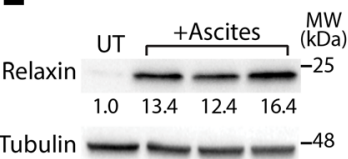

$\mathbf{F} \quad$ LL

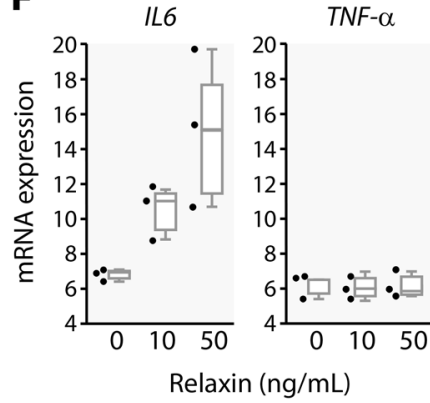

G

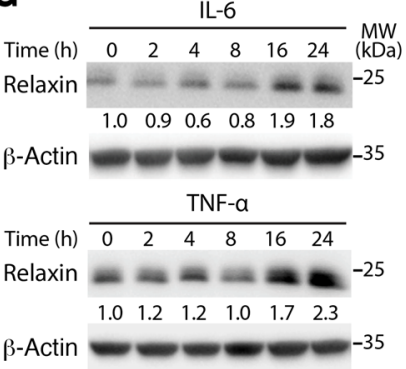

Figure 4. Analysis of relaxin expression in patient-derived OC tumors, serum, and ascites. (A) Relaxin expression in normal ovarian, FT, and OC cell lines. OV4453 is duplicated, as it served as a positive control. Densitometry analysis rounded to the nearest whole number. (B) Relaxin expression (red) in normal FT and HGSOC tissue samples. Original magnification, $\times 8$ (whole core tissue); $\times 15$ (insets). (C) Relaxin levels (pg/mL) in sera derived from patients with epithelial OC $(n=48)$ and healthy donors (normal, $n=14)$. For this and subsequent panels, box plots indicate the IQR of the data and the central line shows the median. ${ }^{* *} P=0.01$, Student's $t$ test. (D) Relaxin $(\mathrm{pg} / \mathrm{mL}$ ) in patient-derived ascites compared with tissue culture media $(M) . n=3$. (E) Analysis of relaxin expression in FT194 cultured for 72 hours in media containing $10 \%$ ascites (+Ascites). (F) IL- 6 and TNF- $\alpha$ mRNA levels following treatment of OVCAR8 with RLN2 $(50 \mathrm{ng} / \mathrm{mL}$ ) for 24 hours. $n=3$. (C) Analysis of relaxin expression in OVCAR8 treated with IL-6 $(50 \mathrm{ng} / \mathrm{mL})$ or TNF- $\alpha(50 \mathrm{ng} / \mathrm{mL})$. (H) Analysis of relaxin expression in OVCAR8 and SKOV3 treated for 24 hours with increasing doses of IL-6 (ng/mL).

nism (Supplemental Figure 4G). Therefore, inflammatory cytokines may promote $\mathrm{OC}$ tumor progression through the induction of RLN expression.

The RLN promoter is regulated by cytokine-induced activation of STAT3 and NF- $\kappa B$. To understand the mechanism of RLN regulation by inflammatory cytokines, the promoters of RLN1 and $R L N 2$ were examined. A series of genomic fragments surrounding the RLN2 transcription start site (TSS) was cloned into a pGL3 promoter-less luciferase plasmid (Figure $5 \mathrm{~A}$ ). RLN2 was chosen over $R L N 1$ because it is more widely expressed. All constructs were functional in OVCAR8 and SKOV3, as demonstrated by high luciferase activity (Figure 5B). A minimal promoter (RP-3) containing several conserved DNA regions was the smallest fragment displaying robust activity. Consistent with a gene duplication event of relaxin genes, the RLN2 RP-3 sequence was more than $90 \%$ conserved, with the corresponding region in the RLN1 promoter suggesting common transcriptional regulation of these 2 genes (Figure 5C).

To identify transcription factors (TF) regulating $R L N$ expression, the RP-3 sequence was examined using in silico TF-binding prediction (ConSite and Match). Because RLN was induced by both IL- 6 and TNF- $\alpha$, we focused on TFs activated by these cytokines, STAT3 and NF- $\mathrm{kB}$, respectively. RP-3 contained several predicted STAT3 and NF- $\mathrm{kB}$ elements, 2 of which mapped to peaks of high conservation (Figure 5, A and C). A highly conserved binding site for SOX9, a protein of the high-mobility group DNA-binding domain family, was also present. Notably, the highly conserved STAT3 and SOX9 binding sites are present in the RLN1 promoter (Figure 5C and Supplemental Figure 5A).

Knockdown of STAT3 in multiple cells diminished luciferase expression from the RP-3 reporter (Figure 5D and Supplemental Figure 5B). Knockdown of SOX9 decreased luciferase expression 
A

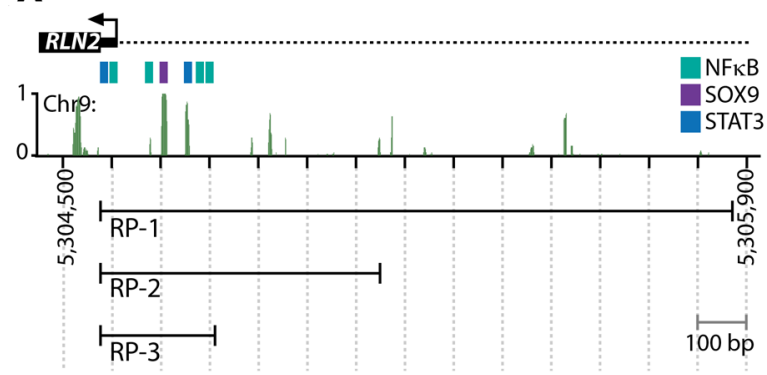

B

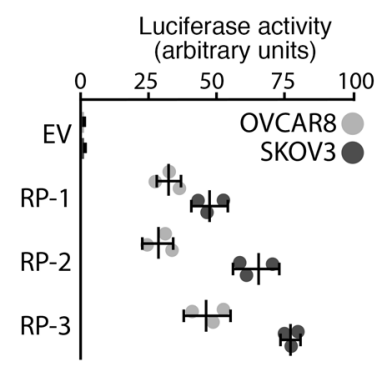

C

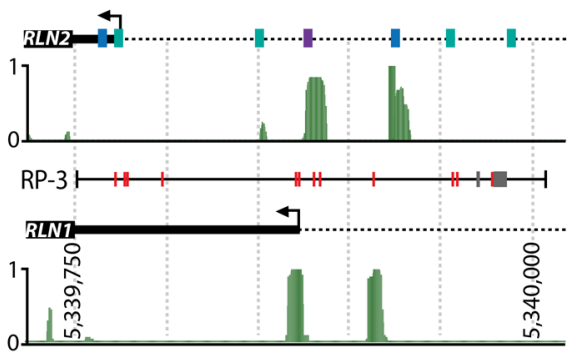

D

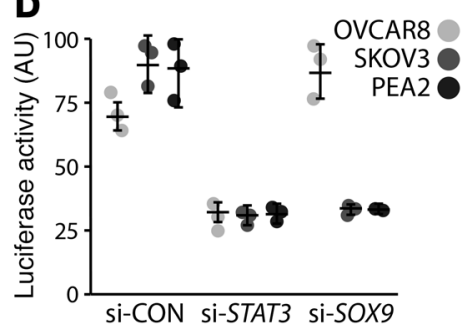

$E$

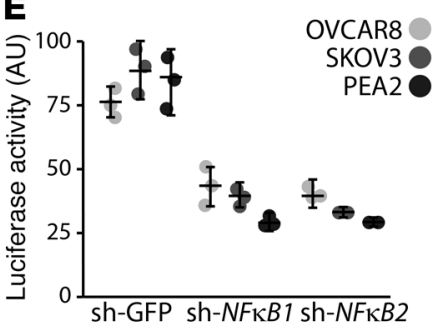

$\mathbf{F}$

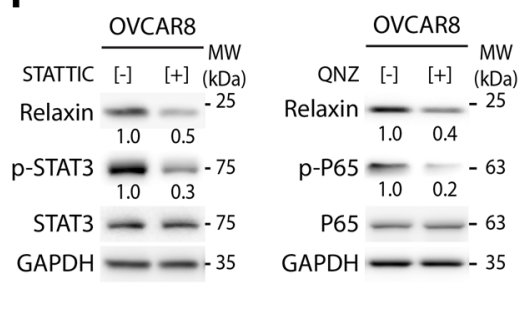

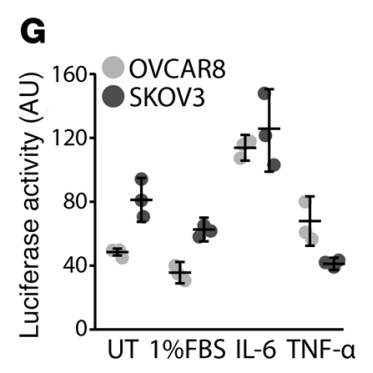
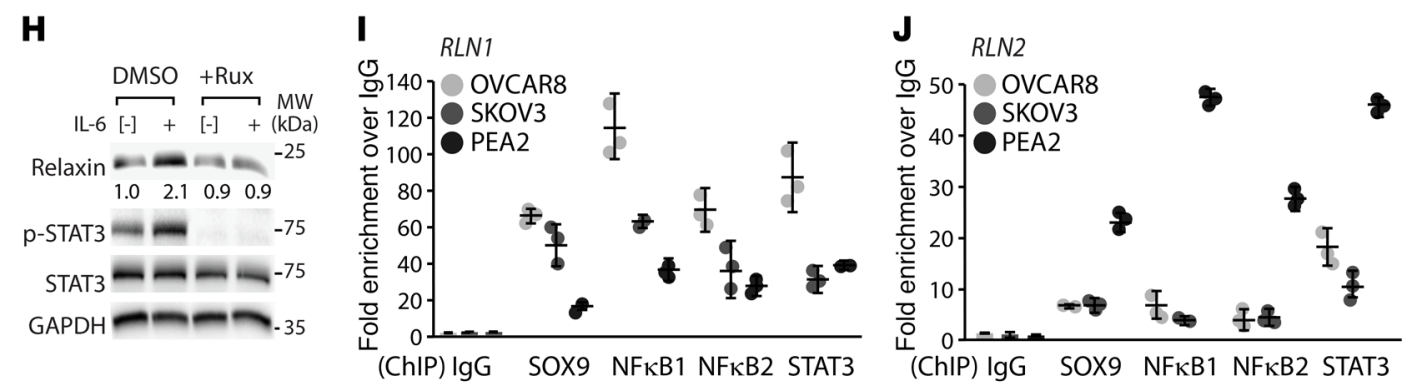

Figure 5. The relaxin promoter is activated by STAT3 and NF-кB. (A) Schematic of the genomic region proximal to the RLN2 transcriptional start site (UCSC genome browser-human GRCh37/hg19). Species conservation is indicated. Boundaries of 3 relaxin promoter (RP) constructs, RP-1, RP-2, and RP-3, are mapped. Predicted binding sites for STAT3, NF- $\kappa B$, and SOX9 are indicated. (B) Luciferase activity of the indicated RP constructs compared with empty vector control (EV) in OVCAR8 and SKOV3. Luciferase activity is normalized to Renila activity. For this and subsequent experiments, error bars indicate mean \pm SEM. $n=3$. (C) Genomic region of the RLN2 promoter (RP-3) compared with the RLN1 promoter. Peaks indicate species conservation. Red bars in the RP-3 sequence indicate single nucleotide differences in $R L N 1$ compared with $R L N 2$, and the open box indicates a small sequence not present in $R L N 1$. Predicted binding sites for STAT3, NF- KB, and SOX9 are indicated. (D) RP-3 luciferase activity in cells transfected with control siRNA (siCON) or siRNA targeting STAT3 or SOX9. $n=3$. (E) RP-3 luciferase activity in cells expressing shGFP or hairpins targeting $N F \kappa B 1$ or $N F \kappa B 2$ subunits (sh-NFKB1 and sh-NFKB2). $n=3$. (F) Relaxin expression and STAT3 phosphorylation (pY705) in OVCAR8 treated for 48 hours with small molecule inhibitors of STAT3 (STATTIC, $1 \mu \mathrm{M})$ or NF- $\kappa \mathrm{B}$ (QNZ, 5 nM) compared with mock-treated (-) cells. (G) RP3-luciferase activity in OVCAR8 and SKOV3 treated with 1\%FBS, IL-6 (50 ng/mL), or TNF- $\alpha$ ( 50 ng/ $\mathrm{mL}$ ) for 24 hours compared with untreated cells. $n=3$. (H) Relaxin levels and STAT3 phosphorylation ( $p$ Y705) in 0VCAR8 treated with IL-6 (50 ng/mL) or control (-) 24 hours after treatment with the JAK1/2 inhibitor Ruxolitinib (+Rux) compared with DMSO. (I and J) ChIP analysis of TF occupancy at the RLN1 promoter (I) and RLN2 promoter (J). ChIP signals are shown as fold enrichment over IgG. $n=3$.

in SKOV3 and PEA2, but no change was observed in OVCAR8 (Figure 5D). Knockdown of $N F \kappa B 1$ or $N F \kappa B 2$ decreased luciferase activity in OVCAR8, SKOV3, and PEA2 (Figure 5E). In OVCAR8, RLN1 mRNA was decreased with TF knockdown (Supplemental Figure 5C). STAT3 or NF- $\mathrm{BB}$ inhibition decreased relaxin expression in OVCAR8 concomitant with decreased phosphorylation of STAT3 and NF- $\mathrm{KB}$ subunit RELA, respectively (Figure 5F).

Consistent with IL-6-induced STAT3 activation (41), RP-3 luciferase activity was significantly increased by IL- 6 treatment in both OVCAR8 and SKOV3 (Figure 5G). TNF- $\alpha$ stimulation activated the RP-3 reporter in OVCAR8, but not SKOV3. JAK1/2 or $\mathrm{NF}-\kappa \mathrm{B}$ inhibition blocked the IL- $6-$ and TNF- $\alpha$-induced relaxin in OVCAR8, confirming cytokine activation of TFs driving $R L N$ expression (Figure 5H and Supplemental Figure 5D). Furthermore, application of ascites fluid to FT cell lines induced phos- phorylated STAT3 (p-STAT3) levels consistent with ascites having high levels of IL-6 (Supplemental Figure 5E). To demonstrate that IL-6 is driving induction of relaxin, IL-6 neutralizing antibody was added to ascites and relaxin expression was suppressed (Supplemental Figure 5F). These results demonstrated that IL-6 in ascites induced RLN expression in OC cells through JAK/STAT pathway activation. Interestingly, 12 of 16 genes in the relaxin gene signature have a STAT3-binding element in their promoter, further supporting relaxin autocrine signaling (Supplemental Figure 5H).

To validate occupancy of TFs on the endogenous $R L N$ pro-

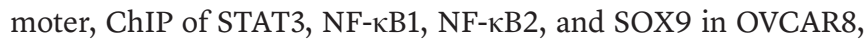
SKOV3, and PEA2 was performed. Distinct amplicons for ChIP were designed at the proximal promoters of both RLN1 and RLN2. Binding of STAT3, NF- $\kappa$ B1, NF- $\kappa$ B2, and SOX9 were enriched on the $R L N 1$ promoter in all 3 cell lines (Figure 5I). IL- 6 treatment 
increased binding of STAT3, while TNF- $\alpha$ treatment increased binding of STAT3, NF- $\kappa \mathrm{B} 1$, and SOX9 on the RLN1 promoter in OVCAR8 (Supplemental Figure 5I). Interestingly, binding of

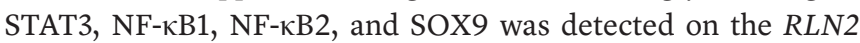
promoter in PEA2 cells, which express both RLN1 and RLN2 relative to OVCAR8, and SKOV3 cells, which express predominantly RLN1 (Figure 5J). In summary, STAT3, NF- $\mathrm{BB}$, and SOX9 are transcriptional activators of $R L N$ in multiple cell lines that act downstream of inflammatory cytokines (Supplemental Figure 5J).

The relaxin/RXFP1 autocrine loop contributes to cisplatin resistance via RLN promoter activation. Activation of cytokine signaling can counteract chemotherapy and promote resistance (35). Since IL- 6 and TNF- $\alpha$ are induced following cisplatin treatment and contribute to chemoresistance (42), we investigated whether relaxin/RXFP1 signaling contributes to cell survival following cisplatin treatment. RXFP1-dependent cell lines tended to be inherently more cisplatin resistant, as determined by the cisplatin $\mathrm{IC}_{50}$ value (Figure $6 \mathrm{~A}$ ). The median cisplatin $\mathrm{IC}_{50}$ value was higher in RXFP1-dependent cell lines versus independent cell lines $(12.3 \mu \mathrm{M}$ versus $5.2 \mu \mathrm{M}, P=0.02$ ). In addition, relaxin was significantly elevated in 9 of 12 patient serum samples following treatment with taxol/carboplatin (Supplemental Figure 6, A and B), consistent with the idea that chemotherapy can induce relaxin expression in OC tumors as a survival adaption to the cytotoxic effects.

Increased IL- 6 and TNF- $\alpha$ were measured in conditioned media from OVCAR8 and SKOV3 and activation of STAT3 and NF- $\mathrm{BB}-\mathrm{P} 65$ was measured in OVCAR and SKOV3 cell lysates following treatment with cisplatin (Supplemental Figure 6, C-E). Consistent with increased activation of relaxin regulating TFs following cisplatin treatment, RP-3 promoter activity and secretion of relaxin were increased in response to cisplatin exposure in both OVCAR8 and SKOV3 (Supplemental Figure 6, F and G).

We reasoned that if induction of RLN is part of an adaptive response of OC cells to cisplatin, then depletion of RLN may increase cisplatin sensitivity. In SKOV3 and PEA2, knockdown of either RXFP1 or RLN combined with sublethal doses of cisplatin significantly affected cell growth compared with shGFP control (Figure 6B). Clonogenic colony assays revealed dramatically decreased colony formation upon treatment with a sublethal dose $(2.5 \mu \mathrm{M})$ of cisplatin in combination with $R X F P 1$ or RLN knockdown (Figure 6C). Analysis of the coefficient of drug interaction (CDI) (43) revealed a synergist effect of RXFP1 or RLN knockdown combined with cisplatin treatment (SKOV3 CDI $<0.27$ and PEA2 CDI $<0.33$ for sh1-RXFP1 or sh1-RLN and $2.5 \mu \mathrm{M}$ Cis; see Methods).

We next tested to determine whether RXFP1 knockdown sensitized tumor cells to cisplatin in vivo. Luciferase expressing OVCAR8 was derived that contained TET-inducible sh1-RXFP1 or shGFP and was injected into the peritoneal cavity of NSG mice (Figure 6D). Two weeks following injection, mice were divided into sucrose alone or sucrose + Dox groups to induce shRNA expression. Three weeks after injection, mice were divided into cisplatin-treated ( $1 \mathrm{mg} / \mathrm{kg}$ once per week for 3 weeks) or vehicletreated groups (Figure 6D). The bioluminescence intensity in the TET-shGFP group with or without Dox increased rapidly and consistently from injection to week 5.5 (Figure 6, E and F). Cisplatin-treated TET-shGFP groups with or without Dox demon- strated slightly reduced tumor growth over the course of the experiment. Consistent with the TET-shGFP control group, the noninduced TET-sh-RXFP1 tumors grew with similar kinetics (Figure 6, E and F). However, induction of TET-sh-RXFP1 knockdown resulted in diminished tumor growth and near tumor eradication in the presence of cisplatin (Figure 6, E-G). The combined effect of RXFP1 knockdown and cisplatin treatment in vivo demonstrated a CDI equal to 0.1 compared with a CDI equal to 0.8 for the shGFPand cisplatin-treatment control group. These results demonstrate potent sensitization of in vivo cisplatin-induced cytotoxicity by inhibition of RXFP1 expression.

Relaxin neutralizing antibody decreases OC viability and potentiates cisplatin cytotoxicity. We hypothesized that relaxin neutralization would be deleterious to OC cell growth and potentiate cisplatin cytotoxicity. RLN1 and RLN2 peptides share $87 \%$ sequence identity within the primary receptor binding (B-chain) domain and $63 \%$ identity within their secondary receptor binding (A-chain) domain. A library of monoclonal antibodies (RLN2Am34) was generated aiming to identify clones that neutralize both RLN1- and RLN2-mediated cAMP induction. Supernatants derived from the RLN2Am34 library identified clones with strong binding to RLN2 and relatively weaker binding to RLN1 (Supplemental Figure 7A). Several clones bound both ligands and were tested for neutralization of relaxin in cell-based cAMP assays (Supplemental Figure 7, B and C). Hybridoma clone m34-21 bound to both RLN1 and RLN2 and demonstrated potent RLN2 neutralization with favorable binding kinetics and a Kd in the low nM range (Supplemental Figure 7D). m34-21 reduced growth and viability of OVCAR8 in a dose-dependent manner, but had no effect on OVCAR5 compared with monoclonal IgG1א isotype control (Figures 7, A-C).

To determine whether neutralization of relaxin by m34-21 increases cisplatin sensitivity, OVCAR8 and OVCAR5 were treated with or without sublethal doses of cisplatin $(1.75 \mu \mathrm{M})$ in combination with m34-21 (50 $\mu \mathrm{g} / \mathrm{mL})$. Cotreatment blocked proliferation in cell culture over a 7-day period compared with OVCAR8 treated with only sublethal cisplatin or sublethal cisplatin plus isotype antibody control (Figure 7D). OVCAR5 showed no further growth impairment from m34-21 in combination with cisplatin.

To establish whether any resistant cells were present after the 7-day treatment, cells were replated for colony formation assays following treatment with cisplatin or with the combined treatment of m34-21/cisplatin (Figure 7E). In OVCAR8, cisplatin or m3421 treatment alone significantly reduced colony formation upon replating (Figure 7F). Strikingly, the combination of m34-21 and cisplatin completely abolished colony repopulation. A CDI equal to 0.34 for OVCAR8 treated with both $\mathrm{m} 34-21$ and cisplatin versus a CDI equal to 0.98 for the isotype control was calculated. OVCAR5 were not sensitive to any treatment (CDI >1.0 for all conditions). Therefore, neutralization of relaxin increased cisplatin sensitivity in RXFP1-dependent OC cells.

\section{Discussion}

The emergence of autocrine loops is a strategy frequently exploited by tumors to proliferate autonomously, establish metastatic programs, and adapt to the cytotoxic effects of chemotherapeutic drugs. Examples of autocrine signaling loops in OC include PDGF-PDGFR, LPA-LPAR, FGF-FGFR, and FSH-FSHR ligand- 
A

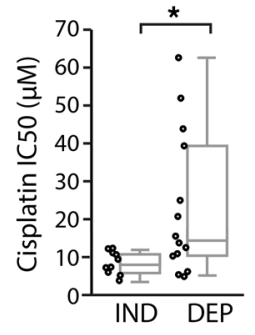

B

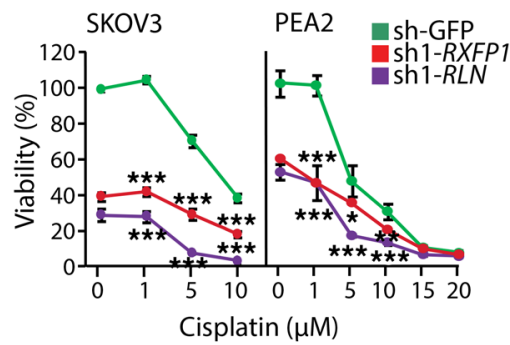

C

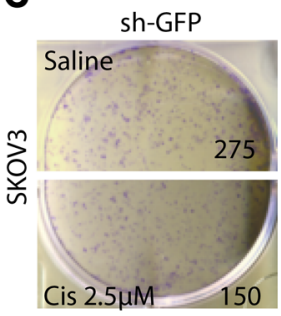
sh-GFP

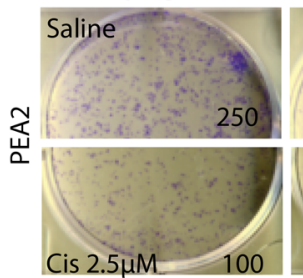

sh1-RXFP1

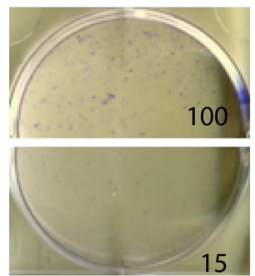

sh1-RXFP1

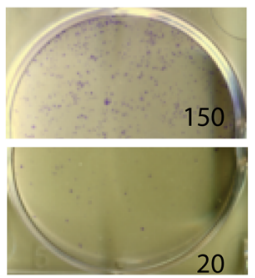

sh1-RLN

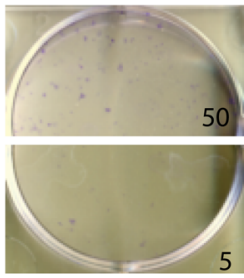

sh1-RLN

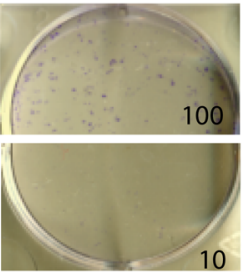

D

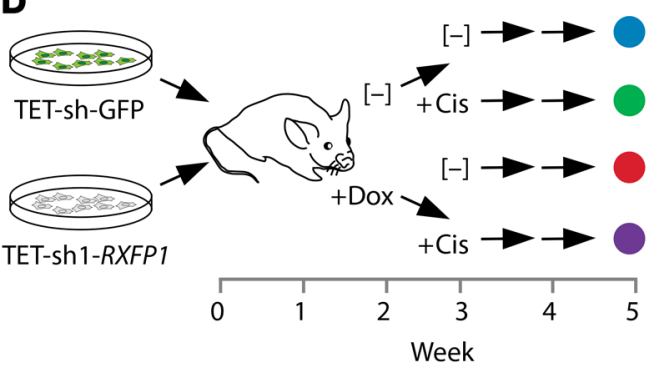

E

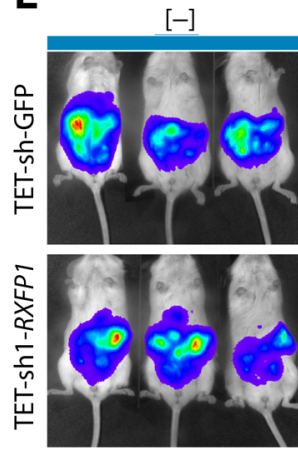

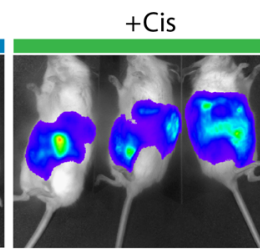

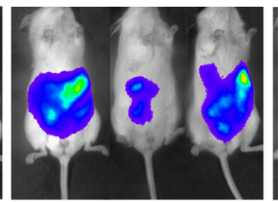

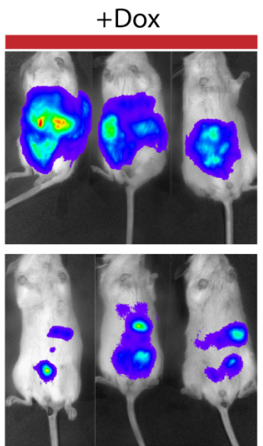

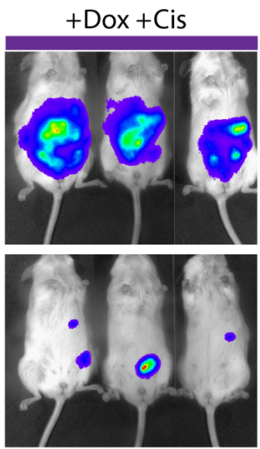

F

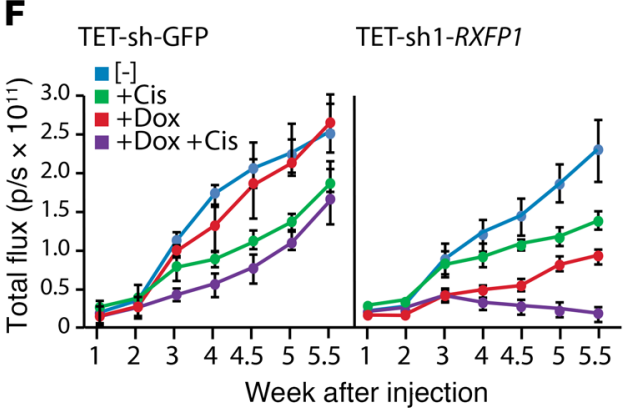

G

TET-sh-GFP
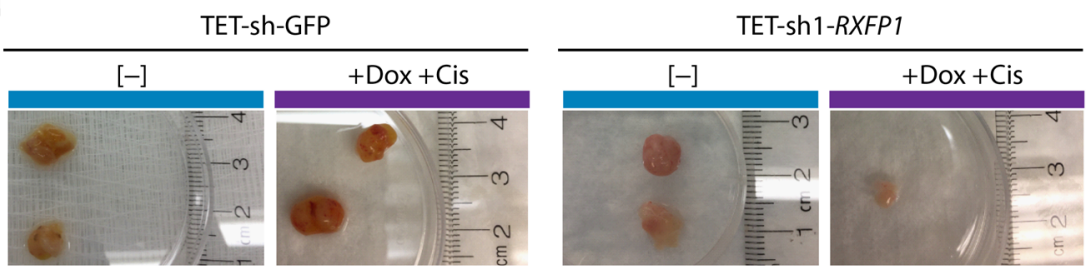

Figure 6. RXFP1 and relaxin knockdown sensitizes HGSOC cells and tumors to cisplatin. (A) Cisplatin IC $\mathrm{C}_{50}$ values $(\mu \mathrm{M})$ in $R X F P 1$-independent (IND, $n=10$ ) and $R X F P 1$-dependent (DEP, $n=14$ ) cell lines. Box plots indicate the IQR of the data, and the central line shows the median. ${ }^{*} P<0.00578$, Wilcoxon's rank test, critical $U=48$ at $P<0.5$. (B) Viability of SKOV3 and PEA2 expressing shGFP control or shRNA targeting RXFP1 (sh1-RXFP1) or relaxin (sh1-RLN) and treated with increasing doses of cisplatin $(\mu \mathrm{M}) .{ }^{*} P<0.03$; ${ }^{* *} P<0.002 ;{ }^{* *} P<0.001$, Dunnett's test. Red and purple lines are compared with the green line. (C) Clonogenic assay of cells expressing shGFP control or sh1-RXFP1 sh1-RLN in the absence (saline) or presence of a sublethal dose of cisplatin (2.5 $\mu \mathrm{M})$. Quantification of colonies per images indicated. (D) Schematic for testing combined effects of Dox-induced RXFP1 knockdown and cisplatin treatment in vivo. Luciferase expressing OVCAR8 coexpressing TET-inducible shRNA control (TET-shCFP) or TET-inducible shRNA targeting RXFP1 (TET-sh1-RXFP1) were injected into the interperitoneal cavity of NSG mice. At week 2, mice were treated with sucrose control (-) or Dox (+Dox) to initiate shRNA expression. At week 3 , mice were divided into untreated (-) or cisplatin-treated (+Cis, $1 \mathrm{mg} / \mathrm{kg}$ per week), which was continued for 3 weeks. Colored dots at the end point of the experiment mark the conditions: blue (-), green (-Dox, +Cis), red (+Dox, -Cis), and purple (+Dox, +Cis). (E) Bioluminescence images of mice bearing intraperitoneal xenografts of OVCAR8 expressing TET-shCFP or TET-sh1-RXFP1 in treatment groups 5 weeks after injection. (F) Quantification of bioluminescence from xenografts expressing TET-shGFP or TET-sh1-RXFP1. Luminescence measurements expressed as total flux (photons/s). Error bars indicate mean \pm SEM. $n=3$. (C) Representative tumors of OVCAR8 expressing TET-shCFP or TET-sh1-RXFP1 extracted at the experimental endpoint showing the blue (-) and purple (+Dox, +Cis) groups. 
A

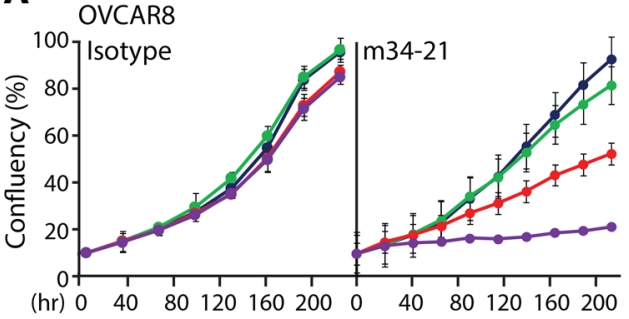

OVCAR5

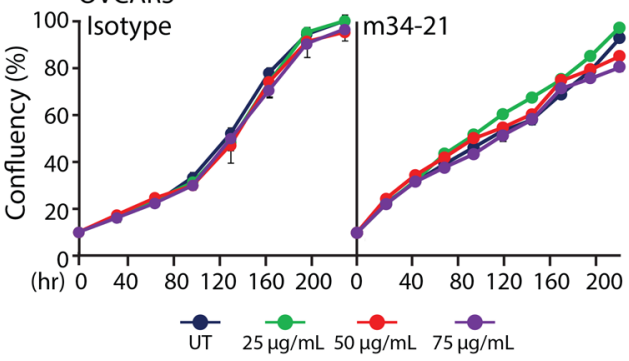

B

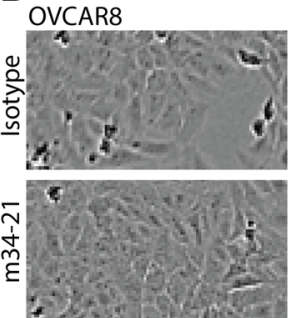

UT

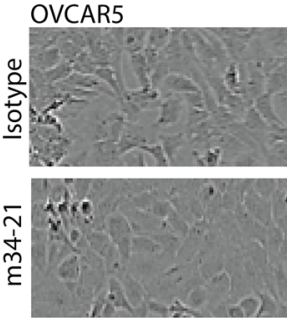

UT

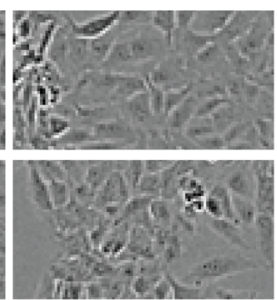

$25 \mu \mathrm{g} / \mathrm{mL}$

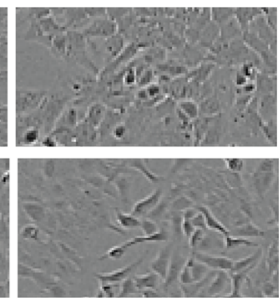

$25 \mu \mathrm{g} / \mathrm{mL}$

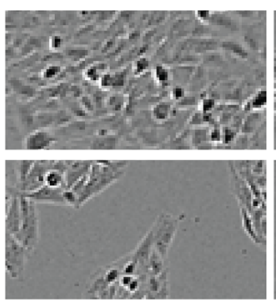

$50 \mu \mathrm{g} / \mathrm{mL}$

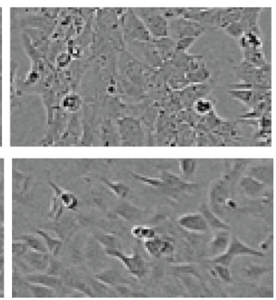

$50 \mu \mathrm{g} / \mathrm{mL}$

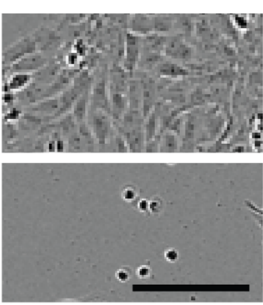

$75 \mu \mathrm{g} / \mathrm{mL}$

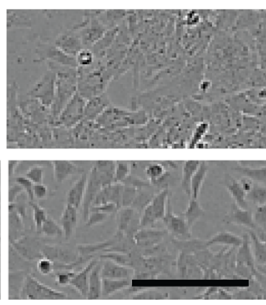

$75 \mu \mathrm{g} / \mathrm{mL}$

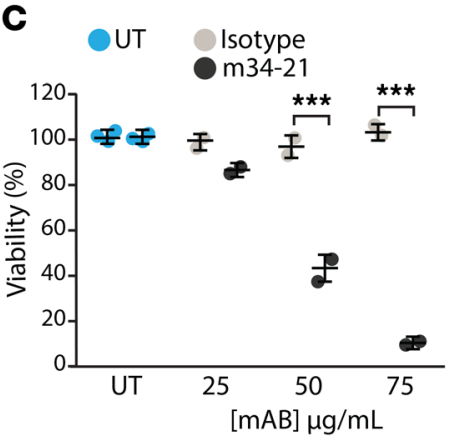

$\mathbf{E}$

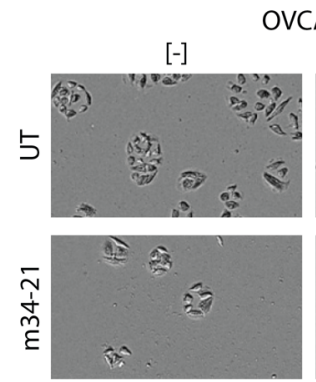

D
OVCAR8

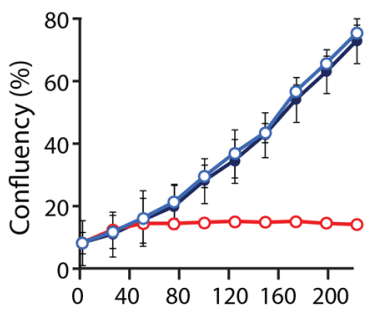

(h)
OVCAR5

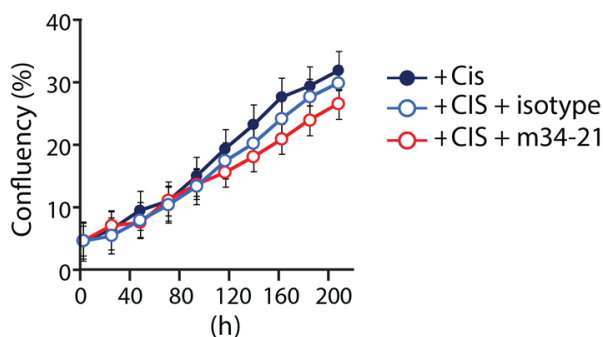

(h)

$\mathbf{F}$

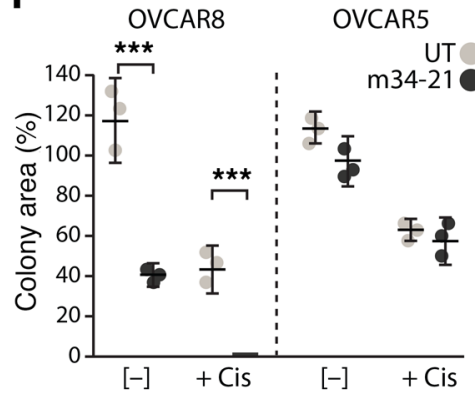

Figure 7. Relaxin neutralizing monoclonal antibody abrogates HCSOC cell growth. (A) Growth of OVCAR8 and OVCAR5 treated with relaxin-neutralizing monoclonal antibody (m34-21) or isotype control. Error bars indicate mean \pm SEM. $n=5$. (B) Representative images of OVCAR8 and OVCAR5 treated with m34-21 or isotype control for 7 days. Scale bar: $10 \mu \mathrm{m} . n=5$. (C) Viability of OVCAR8 treated with m34-21 or isotype control for 7 days. For panels $\mathbf{C}$ and $\mathbf{F}$, box plots indicate the IQR of the data, and the central line shows the median. $n=5 .{ }^{* *} P<0.001$, Student's $t$ test. (D) Growth of OVCAR8 and OVCAR5 treated with a sublethal dose of cisplatin $(1.75 \mu \mathrm{M})$ alone or in combination with m34-21 or isotype control $(50 \mu \mathrm{g} / \mathrm{mL})$. $n=5$. (E) Repopulating colonies formed following no treatment (-) or treatment with cisplatin (+Cis) alone (UT) or in combination with m34-21 (50 $\mu \mathrm{g} / \mathrm{mL})$. Scale bar: $10 \mu \mathrm{m}$. $n=5$. (F) Quantification of the repopulating colony assay as described in E. Quantification was performed using ImageJ $(\mathrm{NIH}) .{ }^{* *} P<0.001$, Student's $t$ test. $n=5$.

receptor pairs (44-47). Here, we report the identification of a relaxin/RXFP1 autocrine loop essential in sustaining survival and proliferation in a subset of OC cells.

Relaxin protects against apoptosis through the activation of multiple signaling pathways, including MAPK and AKT pathways. Transcriptome profiling revealed genes and pathways upregulated by relaxin that collectively sustain proliferation and cancer progression. Top enriched pathways included MAPK signaling, extracellular matrix receptor interaction, NOTCH, and VEGF signaling. Several RLN-upregulated genes encode for secreted factors, including macrophage migration inhibitory factor (MIF), MMPs (MMP9, MMP23B, and MMP15), and $V E G F$, that have potential to influence other cells in the tumor microenvironment. NOTCH1 and NOTCH3 were upregulated following relaxin treatment, supporting emerging studies of the association between relaxin and Notch signaling. Considerable 
evidence supports an important oncogenic role of Notch signaling in HGSOC. Perturbation in the regulation of Notch-1 and Notch-3 as well as Notch ligands has been described, and this has been linked to tumor initiation and progression, metastasis, stemness, and chemotherapy resistance $(48,49)$. The involvement of relaxin/RXFP1 in aberrant regulation of Notch signaling in OC is therefore an important topic of future studies. Relaxin thus induces a transcriptional program that likely contributes to the survival, invasiveness, and overall fitness of OC.

Inflammatory cytokines such as IL- 6 and TNF- $\alpha$ are central mediators of tumor progression in OC. IL- 6 has a central role in OC carcinogenesis and progression through its ability to stimulate invasion of cancer cells through increased expression of MMPs, stimulate the cell cycle, and promote epithelial-to-mesenchymal transition $(50,51)$. High levels of IL- 6 in the serum and ascites of cancer patients produced by monocytes/macrophages and malignant cells is associated with worse clinical outcome and peritoneal metastasis and may play a key role in chemoresistance. Activation of STAT3 is reported to be an early event in the initiation of tumorigenesis within the FT epithelium and to contribute to molecular changes that allow cells to survive in the presence of DNA damage (52). Our results show that $R L N$ is a direct transcriptional target of IL- 6 and TNF- $\alpha$ through the downstream TFs STAT3 and NF- $\kappa$ B. The presence of relaxin in the FT epithelium and its regulation by IL-6/STAT3 coupled with its role in upregulating MMPs and survival pathways suggest that relaxin signaling could contribute to the downstream effects of IL-6/STAT3 in tumor promotion.

We identified a highly conserved SOX9-binding site in the proximal promoters of RLN1 and RLN2 and demonstrated SOX9 occupation on these promoters by ChIP. SOX9 promotes transcription of RXFP2 in human embryonic fibroblast 293T cells and primary rat gubernacular cells (53). Thus, SOX9 may have a more widespread role in the regulation of relaxin-related peptides and receptors in reproductive development and disease. SOX9 is elevated in many types of cancer, including lung, skin, brain, and pancreatic cancers, and high expression correlates with disease progression, chemoresistance, and poor patient survival $(54,55)$. The complex role of RLN regulation by SOX 9 will be an important topic for future study.

Cisplatin resistance is a net effect of multiple mechanisms that either trigger activation of prosurvival pathways or inhibition of cell death pathways (56). Several microenvironmentregulated signaling pathways mediate chemoresistance in OC, including AKT, NF- $\kappa$ B, and STAT3 pathways (57). Elevated IL-6 levels in serum and ascites of EOC patients correlates with the emergence of chemoresistance, although the underlying mechanisms of IL-6-mediated chemoresistance in OC cells are not completely understood. However, some studies showed that IL-6 is associated with increased expression of multidrug resistancerelated genes and apoptosis inhibitory proteins (BCL2, BCLXL, and XIAP) as well as activation of MAPK and PI3K/AKT signaling (58). Here, we identify relaxin/RXFP1 signaling as a prosurvival mechanism induced by cisplatin treatment. We demonstrated that relaxin expression is increased following exposure to sublethal concentrations of cisplatin. In agreement with previous studies, cisplatin treatment also promoted increased secretion of both IL- 6 and TNF- $\alpha$.
Elevated expression of RLN protein was observed in several samples from OC patient serum, tumors, and ascites. Our results corroborate a recent study that measured higher serum relaxin levels in patients with epithelial OC versus those with benign ovarian diseases and healthy controls (59). Serum relaxin was associated with adverse prognosis, with increased levels correlating with International Federation of Gynecology and Obstetrics (FIGO) stage, metastasis, survival, and chemoresistance. We detected increased relaxin levels in sera derived from HGSOC patients as well as patients with clear-cell carcinomas and other OCs. This is consistent with our screening data across our panel of OC cell lines. Although the large majority of lines in this panel were derived from HGSOC, several lines of different histological subtype also demonstrated dependency on RXFP1.

Our results demonstrate that targeting the relaxin/RXFP1 pathway may have therapeutic potential for treating a subset of OC patients, particularly in combination with standard platinum therapy, and could potentially overcome chemoresistance of platinum-resistant tumors.

\section{Methods}

Source of primary samples. HGSOC tissue micro-array (TMA) constructed from formalin-fixed paraffin-embedded tissues was obtained from Centre Hospitalier de l'Université de Montreal. HGSOC tumors from 73 female patients were added to the TMA. Patients had no neoadjuvant chemotherapy prior to sample collection at surgery. Nine cases of normal FT tissues from women without gynecological malignancy were added to the TMA as controls. Serum samples used for Figure 4C were obtained from the Penn Ovarian Cancer Research Center-BioTrust Collection. Serum samples used for Supplemental Figure 6, A and B, were obtained from Centre Hospitalier de l'Université de Montreal.

Antibodies. The following antibodies were used: AbCam relaxin (catalog ab183505); AKT (catalog 9272), p-AKT (catalog 4056, 4058), BCL2 (a), caspase-3 (catalog 9662), cleaved caspase-3 (catalog 9664), GAPDH (catalog 2118), MEK (catalog 4694), p-MEK (catalog 9154), PARP (catalog 9532), cleaved PARP (catalog 9548), STAT (catalog 9139), and p-STAT (catalog 9145) (Cell Signaling Technologies), all at 1:1000 in 5\% milk/PBST buffer; $\beta$-actin (catalog sc-8432), TUBULIN (catalog sc-69969) (Santa Cruz Biotechnology Inc.) at 1:3000.

Anti-relaxin $m A B$ cell treatments. Cells were plated in 96-well plates at 3000 cells/well in RPMI (2\% FBS). Immediately following plating, purified anti-relaxin monoclonal antibodies were added to each well at the indicated concentrations. Cell growth was monitored using IncuCyte (Essen BioScience). For experiments testing combined treatment of anti-relaxin monoclonal antibodies and cisplatin (MilliporeSigma p4394), cells were plated as described above in the absence or presence of cisplatin $(1.75 \mu \mathrm{M})$ and $\mathrm{mAB}$ m34-21 $(50 \mu \mathrm{g} / \mathrm{mL})$. Cells were replated from 96 wells to 12 wells for repopulation assay following 7-day cisplatin exposure. Repopulating colonies were fixed in $4 \%$ paraformaldehyde and stained using $0.005 \%$ $\mathrm{w} / \mathrm{v}$ crystal violet/70\% ethanol. Plates were scanned and quantified using Image J Colony Area Plug-in (NIH).

Anti-relaxin monoclonal antibodies. To generate monoclonal antibodies against RLN2, 4 female BALB/c mice were immunized in a single hind footpad with synthetic RLN2 (Phoenix Peptide) in Ribi Adjuvant (MilliporeSigma Adjuvant System). Mice received booster 
injections (with adjuvant) twice weekly for 4 weeks. Serum was collected on day 21 to check titers by ELISA against RLN2 and RLN1; all mice showed a strong serum response. Mice received a final boost of RLN2 (PBS, no adjuvant) on day 28. Splenocytes and lymphocytes from mice were harvested on day 31. A hybridoma library (BALB/c RLN2Am34) was created by PEG fusion of the pooled cells with P3X63Ag8.653 (CRL-1580) myeloma cells. After bulk HAT selection, the hybridoma library was cryopreserved.

Ascites fluid processing. Fluid was collected from HGSOC patients and processed within 24 hours. Fluid was centrifuged at $300 \mathrm{~g}$ for 10 minutes at $4^{\circ} \mathrm{C}$. To disrupt large clusters of cells/spheroids, pellets were suspended in PBS and filtered through sterile butter muslin; clusters greater than $70 \mu \mathrm{m}$ were isolated. Isolated clusters were then dissociated with TrypLE Express (Invitrogen).

Biolayer interferometry for kinetics. Binding kinetics between purified RLN2Am34-M21 (m34-21) antibody and RLN1 and RLN2 were evaluated by biolayer interferometry on the ForteBio Octet Red96. Purified m34-21 antibody was immobilized onto ForteBio AntiMouse-Fc Capture (AMC) biosensors $(5 \mu \mathrm{g} / \mathrm{mL})$, followed by quenching of the AMC biosensors with irrelevant mouse $\operatorname{IgG}(150 \mu \mathrm{g} / \mathrm{mL})$. After a baseline step, real-time measurement of the association and dissociation of RLN1 and RLN2 was performed at 5 concentrations $(111,37.0,12.3,4.12$, and $1.37 \mathrm{nM})$. No significant binding of RLN1 to M21 was observed. On rates $\left(\mathrm{k}_{\mathrm{on}}\right)$, off rates $\left(\mathrm{k}_{\mathrm{off}}\right)$, and the overall molar affinity constant $\left(\mathrm{K}_{\mathrm{D}}\right)$ for $\mathrm{m} 34-21$ binding to each concentration of RLN2 were calculated using a 1:1 model using ForteBio Data Analysis software. A 1:1 Global $\mathrm{K}_{\mathrm{D}}$ fit was also performed across multiple concentrations of each antigen, yielding a calculated $\mathrm{K}_{\mathrm{D}}$ of M21 binding to RLN2 of $7.7 \times 10^{-10}(0.77 \mathrm{nM})$.

$B r d U$ assay. Cells were transfected with RXFP1-targeting siRNAs as described above. Twenty-four hours after transfection, cells were plated in 96-well plates at a density of 5000 cells/well. Cells were serum starved for 16 hours and treated with or without recombinant relaxin for 24 hours ( $n=3$ per group). BrdU incorporation was measured according to the manufacturer's instructions (Cell Signaling Technology, 6813), and absorbance was read at $450 \mathrm{nM}$.

cAMP assay. For detection of cAMP, 5000 cells were seeded in a 96-well plate in RPMI (10\% FBS). Twenty-four hours after seeding, cells were starved for 8 hours, pretreated with $1 \mathrm{mM}$ IBMX (500 $\mu \mathrm{M}$, Stem Cell Technologies, 72762) for 2 hours, and then treated without or without $50 \mathrm{ng} / \mathrm{mL}$ recombinant human relaxin rhRLN2 (Phoenix Pharmaceuticals, 035-62) for 30 minutes. Pelleted cells were suspended in $300 \mu \mathrm{l} 0.1 \mathrm{M}$ HCL for 10 minutes. cAMP levels were determined by ELISA according to the manufacturer's instructions (Enzo Life Sciences, ADI-901-163).

Cell confluence and viability. From 1 to $2 \times 10^{5}$ target cells were infected as indicated. Following selection with puromycin, cells were trypsinized, counted, and plated in 96-well plates at $2.0 \times 10^{3}$ cells/ well. Cell confluence was monitored using an IncuCyte Kinetic Imaging System (Essen BioScience) until shGFP-expressing cells reached confluence. Viability was determined using PrestoBlue (Thermo Fisher Scientific, A13261) according to the manufacturer's protocol.

Cell lines. OVCAR8, SKOV3, PEA1, PEA2, PEO4, PEO6, and OVCAR5 were obtained from the ATCC and cultured in RPMI 1640 with $10 \%$ fetal bovine serum. OAW42 was cultured in DMEM containing 20IU/L bovine insulin and 10\% FBS. Information on all cell lines used for shRNA screening has been previously described (11). FT194 was maintained in DMEM/F12 containing 2\% USG. Normal ovarian surface epithelial cell lines (NOV3198G, NOV3918G+C, NOV3202G, NOV3210, and NOV2309) were established as described previously (60) and maintained in OSE $+10 \%$ FBS. All cultures were maintained at $37^{\circ} \mathrm{C}$ in $5 \% \mathrm{CO}_{2}$. Identity of all cell lines was validated by STR profiling, and each cell line was confirmed negative for mycoplasma.

Cell treatments. Cell lines were starved in RPMI containing 0\% FBS for 16 hours, then treated with human recombinant RLN2 (50 ng/mL, Phoenix Pharmaceuticals, 035-62), hIL-6 (5-100 ng/mL, CST 8904), or hTNF- $\alpha$ (5-100 ng/mL, CST 8902) for the indicated duration. For ascites coculture experiments, cells were starved and then cultured in serum-free media containing $10 \%$ ascites for 72 hours in the absence or presence of $\alpha$ IL- 6 neutralizing antibody (10 $\mu \mathrm{g} / \mathrm{mL}, \mathrm{R} \& D$ Systems AF-206-NA). For STAT3 and NFKB inhibition, cells were treated with Stattic $(1 \mu \mathrm{M}$, Selleckchem S7024) or QNZ (5nM, Selleckchem S4902) for 16 to 48 hours.

Cell-based relaxin ELISA. Following selection for shRNA constructs, 3000 cells were plated in complete media for 24 hours and then plated in serum-free media. After 72 hours, media was collected and protein levels measured by ELISA according to the manufacturer's instructions (Immunodiagnostik, K9210).

ChIP. ChIP was performed using SimpleChIP Enzymatic Chromatin IP kit with magnetic beads (Cell Signaling Technology, 9003) following the manufacturers' protocol. The following antibodies were used: anti-SOX9 (Santa Cruz Biotechnology Inc., catalog sc-166505X), anti-NF-кB1 (Cell Signaling Technology, catalog CST 13586S), antiNF-кKB2 (catalog CST 37359), anti-STAT3 (Cell Signaling Technology, catalog CST 9139S), and control IgG (Abcam, catalog ab18413).

Clonogenic survival assay. Cells transduced with the indicated constructs were plated at 2500 cells/well in 6-well plates. Saline or cisplatin $(1,2.5$, and $5 \mu \mathrm{M}$, MilliporeSigma, p4394) was added 48 hours after plating. Following an additional 48 hours, 2500 cells from each treatment were replated in normal culture media (no cisplatin) and colonies were formed over 2 to 3 weeks. Cells were fixed and stained with $4 \%$ paraformaldehyde and $0.5 \%$ crystal violet in $20 \%$ methanol, then washed and scanned.

CDI. CDI was performed as described (43). Briefly, the CDI is determined by the following formula: $\mathrm{CDI}=(A+B) /(A B)$, where $A$ is the survival percentage of effect or treatment 1 and $B$ is the survival percentage of effect or treatment 2. $A B$ is the survival percentage of the combined effects or treatments. Percentage survival is calculated using Alomar Blue assay, cell counting, or tumor flux as required per experiment using fixed ratio and different concentrations of cisplatin, as indicated in each experiment.

Colony formation. $1.0 \times 10^{3}$ Cells were suspended in $0.5 \mathrm{ml}$ of $0.35 \%$ Bacto agar $(\mathrm{BD}, 214050)$ in growth media and plated on $0.5 \mathrm{ml}$ of $0.5 \%$ Bacto agar base in 12-well plates. The number of colonies $(>50$ cells) was scored after 2 to 3 weeks of incubation by first staining cells with $0.005 \% \mathrm{w} / \mathrm{v}$ crystal violet in $70 \%$ ethanol, then counting the colonies by eye under a light microscope.

Flow cytometry. For apoptosis assays, cells were trypsinized, counted, washed in ice-cold PBS, and suspended in staining media ( $1 \times$ HBSS, $\left.2 \% \mathrm{FBS}, 2.5 \mathrm{mM} \mathrm{CaCl}_{2}\right)$. Cells were transferred to polystyrene tubes, and $1.0 \times 10^{6}$ cells were stained with $100 \mu \mathrm{g} / \mathrm{ml}$ PI (Santa Cruz Biotechnology Inc., 25535-16-4) and $5 \mu$ annexin V-FITC (BD 556419) for 15 minutes at room temperature (RT). Cells were suspended in $400 \mu \mathrm{l}$ staining media, and fluorescence was measured using a 
Becton-Dickinson LSRII flow cytometer. FCS 3.0 files were analyzed using FlowJo, version 9.2.

Hybridoma analysis by ELISA. Mouse sera, polyclonal supernatants from each hybridoma library, and monoclonal hybridoma supernatants after single-cell cloning were analyzed using a coated antigen, anti-IgG reporter ELISA. EIA/RIA 96-well plates (Corning) were coated overnight $\left(4^{\circ} \mathrm{C}\right)$ with either fixed concentrations or serial dilutions of RLN1 (R\&D Systems) or RLN2 (Phoenix Peptide) diluted in $1 \times$ PBS. After blocking with PBS/casein, plates were washed and incubated with serum (1/1000 dilution) or hybridoma supernatant (undiluted), followed by 1/5000 dilution of goat anti-mouse IgG-HRP (Jackson ImmunoResearch), washed, and incubated with TMB Substrate Solution (Moss Substrates) for 30 minutes. ELISA Stop Solution (1M H3PO4) was added to the wells, and absorbance was measured at $450 \mathrm{~nm}$ on an EnSpire microplate reader (PerkinElmer). Incubations were for 1.5 to 2 hours at ambient temperature. To isolate clones from the RLN2Am34 library, viable hybridoma cells were sorted via a BD FACS Aria III, 1 cell per well, into 96-well plates (10-20 plates from each library). After 12 days in culture, monoclonal hybridoma-conditioned supernatants from each 96-well plate were sampled for ELISA screening against RLN2. From ten 96-well plates from the RLN2Am34 library, 41 RLN2-binding clones were identified, and 25 were selected for expansion and cryopreservation.

Hybridoma neutralizing activity analysis. THP-1 cells were suspended at $5 \times 10^{6}$ cells $/ \mathrm{mL}$ in RPMI $(10 \% \mathrm{FBS})$ and pretreated with IBMX for 30 minutes at $37^{\circ} \mathrm{C}$. Pelleted cells were suspended in the absence or presence of $10 \mathrm{ng} / \mathrm{mL}$ recombinant human RLN2 (Phoenix Pharmaceuticals, 035-62) in hybridoma base media (DMEM) or hybridoma clonal supernatants ( $1 \mathrm{~mL}$ total volume) and incubated for 20 minutes at $37^{\circ} \mathrm{C}$. Pelleted cells were suspended in $300 \mu \mathrm{l} 0.1 \mathrm{M} \mathrm{HCL}$ for 10 minutes. cAMP levels were determined by ELISA according to the manufacturer's instructions (Enzo Life Sciences, ADI-901-163).

Immunoblotting. Cell lysates were prepared in RIPA buffer $(50 \mathrm{mM}$ Tris-HCL [pH 7.5], $150 \mathrm{mM} \mathrm{NaCl}, 1 \%$ Triton X-100, 1\% Na deoxycholate) containing $10 \mathrm{mM} \mathrm{NaF}, 1 \mathrm{mM} \mathrm{Na}_{3} \mathrm{VO}_{4}$, and $1 \mathrm{mM}$ PMSF with $1 \times$ Halt Protease Inhibitor cocktail (Thermo Fisher). Lysates were incubated on a rotating plate at $4^{\circ} \mathrm{C}$ for 30 minutes, cleared by centrifugation, and quantified using Pierce BCA Protein Assay Kit (Life Technologies). Cleared lysates were suspended in $2 \times$ sample buffer and boiled; $20 \mu \mathrm{g}$ protein was resolved by SDS-PAGE and transferred to PVDF membranes. For experiments examining phosphorylated protein activation, cells were lysed directly in $2 \times$ sample buffer at the indicated time points. Membranes were blocked in $5 \%$ milk, washed $1 \times$ in PBST, and incubated with antibodies diluted in blocking buffer using standard protocols. Membranes were developed using ECL prime Western blotting detection reagent (GE HealthCare Life Sciences) using the MicroChemi apparatus (DNR Bio-Imaging Systems). Densitometry of Western blots was performed using Quantity One (Bio-Rad) software.

Immunofluorescence. TMA was deparaffinized at $60^{\circ} \mathrm{C}$ for 15 to 20 minutes and washed twice with toluene to remove residual paraffin. Antigen retrieval was performed using the BenchMark XT Ventana Automated Staining system (Ventana Medical Systems). Slides were incubated for 1 hour at $37^{\circ} \mathrm{C}$ with primary antibodies directed against RLN2 (1:500, catalog ab183505, Abcam), washed in PBS, blocked with blocking reagent (Dako), incubated with fluorescent secondary antibodies for 45 minutes at RT, washed in PBS, blocked overnight with Mouse On Mouse Blocking Reagent (MKB-2213, Vector
Laboratories), and incubated with antibodies directed against keratins (1:200, catalog KRT7, MS-1352-P, Neomarkers; catalog KRT18, sc-6259, Santa Cruz Biotechnology; catalog KRT19, MS-198-P, Thermo Scientific) for 1 hour at RT. Slides were quenched with $0.1 \% \mathrm{~m} / \mathrm{v}$ Sudan Black in $70 \%$ ethanol for 15 minutes, washed, and mounted with ProLong Gold Antifade containing DAPI (Molecular Probes, P36935). TMA slides were scanned with a $20 \times 0.75 \mathrm{NA}$ objective with a resolution of $0.3225 \mu \mathrm{m}$ (VS110, Olympus). Fluorescent intensities of RLN2 staining in the epithelial or stromal structures were quantified as MFI using Visiopharm software (Visiopharm). For all markers, MFI dichotomization into high versus low expression was established using ROC curves (SPSS).

Lentivirus. $2.2 \times 10^{5}$ HEK293T cells were cotransfected with 500 ng packaging plasmid pPAX2, 50 ng envelope plasmid VSV-G, and 500 ng of shRNA-expressing pLKO.1 plasmid using XtremeGENE 9 (Roche), according to the manufacturer's protocol. Twenty-four hours after transfection, media was replaced with DMEM (30\% FBS) and cells were incubated for 24 to 48 hours. Lentiviral supernatants were collected, passed through a $0.45 \mu \mathrm{m}$ filter, and stored at $-80^{\circ} \mathrm{C}$. Recipient cells were infected in media containing $6 \mu \mathrm{g} / \mathrm{mL}$ polybrene (MilliporeSigma) for 24 hours and incubated with growth media containing 5-10 $\mu \mathrm{g} / \mathrm{mL}$ puromycin (BioShop) for 48 hours.

Organoids. HGSOC organoid models were generated by the Princess Margaret Living Biobank (PMLB) Organoid Core Facility. Dissociated cells were seeded at a density of 80,000 cells/well in $100 \%$ growth factor-reduced matrigel (VWR) on prewarmed 24-well plates. Solidified domes were overlaid with ovarian growth media (DMEM/F12 supplemented with Glutamax, HEPES, anti/anti, 100 $\mathrm{ng} / \mathrm{mL}$ noggin, $1.25 \mathrm{mM} \mathrm{N}$-acetylcysteine, $1 \mathrm{mM}$ nicotinamide, 100 ng/mL FGF10, 100 ng/mL bFGF, 10 uM Rocki [Y27632], 20 ng/mL EGF, $10 \mathrm{mM}$ Forskolin, $100 \mathrm{nM}$ 17-B-estradiol) and maintained in $37^{\circ} \mathrm{C} 5 \% \mathrm{CO}_{2}$. Cultures were passaged after 14-21 days, with media changes every 3-4 days. Matrigel and cells were dissociated with TrypLE Express for 20 minutes at $37^{\circ} \mathrm{C}$ and passaged at ratios of $1: 1$ to 1:4 into fresh Matrigel. Organoid cultures and PDX model identities were matched to patient tissue by short tandem repeat (STR) analysis. Organoid cultures were routinely tested for Mycoplasma contamination. For IHC, organoid cultures were fixed in PFA and embedded in histogel (Thermo Fisher Scientific). Tumor tissues were embedded in paraffin, sliced into $4 \mu \mathrm{m}$ portions, dried overnight at $60^{\circ} \mathrm{C}$, and stained with antibodies using BenchMark XT autostainer (Ventana Medical Systems). Primary antibodies for IHC analysis were specific to AE1/AE3, p53 (DAKO), Pax8 (ProteinTech Group), and RXFP1 (MilliporeSigma, HPA027067). Slides were imaged using an Aperio Scascope XT (Leica) microscope.

Pathway enrichment analysis. RLN2 upregulated gene set $\left(\log _{2}\right.$ fold change $[\mathrm{FC}]>0.3, P<0.01)$ was processed using g:Profiler (g:Profiler version e94_eg41_p11_88c9db6, database updated on 01/24/2019). Output was subsequently visualized in Cytoscape (version 3.7.1) using the Enrichment Map App. Depmap analysis of the dependency of OC cell lines on the RLN2-regulated gene panel was conducted using the CRISPR (Avana) Public 19Q2 data set. cBioportal for cancer genomics was used to query the alterations of the RLN2-regulated gene panel in clinical tumor samples, and correlation with survival data was obtained from TCGA.

PDX tissue processing. PDX model was generated from ascites collected as indicated above. $2 \times 10^{7}$ Cells were immersed in matrigel 
(10\% Matrigel in FBS-RPMI media) and implanted at the subcu site of NODSCID (PMLB, UHN). PDX tumors were harvested at $1 \mathrm{~cm}$ diameter and processed immediately. The tissue was minced into $1 \mathrm{~mm}^{2}$ pieces, washed with PBS, and enzymatically dissociated with Liberase (MilliporeSigma) for half an hour at $37^{\circ} \mathrm{C}$ with agitation.

Plasmid cloning. Luciferase promoter vectors were cloned from digested PCR products amplified from human gDNA (Roche) into KpnI/XhoI cut pGL3-Basic Vector (Promega). Dox-inducible shRXFP1 or control shRNA constructs were generated by annealing sense and antisense oligonucleotides, followed by phosphorylation with T4 kinase and ligation into the AgeI/EcoRI sites of tet-on-pLKO vector.

Promoter luciferase assay. $7.0 \times 10^{4}$ Cells/24 well were cotransfected with $50 \mathrm{ng}$ pRLN2-Luc or empty vector (pGL3-Basic, Promega) and $1 \mathrm{ng}$ phRL-SV40 (Promega) using Lipofectamine 2000 (Invitrogen) according to the manufacturer's instructions. Twentyfour hours following transfection, cells were lysed and assayed for firefly and Renilla luciferase activity using the Dual-Luciferase Reporter System (Promega).

Quantitative PCR. RNA was extracted from cells using TRIzol (Thermo Fisher) according to the manufacturer's protocol. cDNA was synthesized from 0.5 to $1 \mu \mathrm{g}$ RNA using QuantiTect (QIAGEN). quantitative PCR (QPCR) was performed using Fast SYBR Green Master Mix (Life Technologies) or TaqMan Fast Advanced Master Mix.

RNA-Seq analysis. OVCAR8 were starved in RPMI containing 0\% FBS for 16 hours, then treated with human recombinant RLN2 (50 ng/ $\mathrm{mL}$, Phoenix Pharmaceuticals, 035-62) for 8 hours. RNA was extracted using TRIzol. STAR (version 2.4.2a) was used to quantify transcripts following RNA-Seq, using hy38 as the reference and Gencode (version 25) for annotation. Differential analysis of quantified read counts from across the samples was facilitated by the DESeq2 package (version 1.16.1) in the R statistical environment (version 3.4.1). Transcripts with zero reads mapped across all samples were filtered out prior to downstream analysis. Read counts were then collapsed to gene level, which for the most part resulted in a one-to-one match. In the small fraction of cases where multiple transcripts existed, the transcript with the highest reads mapped was kept. To minimize noise and improve future validation success and efficiency, genes with a low level of expression were filtered out by applying a minimum sum of at least 10 reads mapped in total per gene across the 6 samples. FCs were generated from the filtered count data matrix, modeled as a function of condition (vehicle vs. RLN2 treated) using the DESeq2 package, and $P$ values were further adjusted for multiple testing using a FDR of $1 \%$. Significant hits were defined as genes with an FDR-adjusted $P$ value of at least 0.01 and an absolute $\log _{2}$ FC greater than 1 between conditions.

siRNA. Cells were seeded at $1.0 \times 10^{5}$ cells/well and transfected with $10 \mathrm{nM}$ siGenome SMARTpool targeting RXFP1 (Thermo Fisher, siGENOME SMARTpool, M-005649-01) or a nontargeting siRNA control (Thermo Fisher, siGENOME SMARTpool) or Silencer Select siRNAs targeting RXFP1 (Thermo Fisher, catalog 4392420, assay ID s34026 and s34027) or control siRNAs (Thermo Fisher, catalog 4390843 or 4390846). Cells were transfected using DharmaFECT 1 reagent (Horizon Discovery, T-2001-01), according to the manufacturer's protocol.

Tumor microvessel density. Paraffin-embedded tissues were sectioned $(5 \mu \mathrm{m})$ and rehydrated; antigen retrieval was performed using citrate buffer solution (Abcam,ab93684, Antigen Retrieval Buffer). Heat-induced epitope retrieval was performed for 20 minutes at $98^{\circ} \mathrm{C}$, and then the samples were cooled, washed in tap water for 5 minutes, and blocked with serum-free protein block (Dako, cata$\log$ X0909). Sections were incubated for 1 hour at RT with primary antibody to detect CD31 (1:50, rabbit anti-CD31, Abcam 28364) and stained with biotinylated secondary antibody (anti-rabbit Ig, Vector Laboratories, BA-1000) for 30 minutes at RT. Detection was carried out with the Avidin Biotin Complex system (Vector Laboratories, PK-6100). Sections were incubated in DAB (Abcam, ab64238, DAB Substrate Kit) solution for 10 minutes. Samples were dehydrated, cleared in xylene, and mounted. To assess microvessel density (MVD), CD31-positive clusters were counted at $2 \times 0$ magnification and were normalized to unit area.

Xenograft models. Xenografts were conducted in female NOD/ SCID/IL2R $\gamma^{-/-}$(NSG) mice. $1.0 \times 10^{6}$ Cells in $100 \mu \mathrm{l}$ equal volume matrigel (BD, 354230) and $1 \times$ PBS were injected into the MFP of 3 mice for a total of 6 per condition. Tumor measurements were taken biweekly and continued until tumor size reached $1.5 \mathrm{~cm}$ or tumors became ulcerated. Tumors were removed, weighed, measured, and fixed in $10 \%$ buffered formalin for histology or flash-frozen in liquid nitrogen. Tumor volume (V) was calculated by the formula $V=\pi / 6 \times l$ $\times w^{2}$, where $l$ and $w$ denote the longest and shortest diameters, respectively. For mice treated with Dox, drinking water contained $1 \mathrm{mg} / \mathrm{mL}$ Dox (Bio Basic). Animals in the untreated group were given water with $5 \%$ sucrose. For intraperitoneal xenograft assays, OVCAR8 were stably transduced with PKG-GFP-IRES-Luc vector and sorted for GFPpositive cells by flow cytometry. Cells were assessed for luciferase activity in vitro by treating with media containing $150 \mu \mathrm{g} / \mathrm{ml}$ D-Luciferin (PerkinElmer 122799) for 10 minutes and luminescence measured with the GloMax-Multi Detection System (Promega). Luciferase-expressing cells were prepared for injection as described above. Tumor growth was monitored by injecting $10 \mu \mathrm{l}$ of D-Luciferin/g body weight and bioluminescence imaged using the Xenogen IVIS Spectrum Imaging System (PerkinElmer) in animals anesthetized with 2\% isoflurane. Bioluminescence was quantified using IVIS Live Image software (Caliper Life Sciences).

See complete unedited blots in the supplemental material.

Statistics. A 2-tailed Student's $t$ test or Whitney-Mann $U$ test was used to test significance in experimental conditions. Pairwise comparisons between treatment groups and a single control group were performed using Dunnett's test from the R package DescTools (version 0.99.38). $P$ values of less than 0.05 were considered significant.

Data availability. The RNA-Seq data set produced in this study was deposited in the NCBI's Gene Expression Omnibus database (GEO GSE151280.

Study approval. Written, informed consent was obtained from all patients prior to sample collection and prior to inclusion in this study. Participants are not identified by name. For human samples, ethical approval was obtained from the (CHUM) Institutional Ethics Committee (Comité d'Éthique de la Recherche du CHUM). Experimental mice received environmental enrichment. Animal rooms were maintained at 20 to $24^{\circ} \mathrm{C}, 40 \%$ to $65 \%$ humidity, and a 12 -hour light/12-hour dark cycle. All animal studies were approved by the Animal Research Council of the UHN.

\section{Author contributions}

HEB and RR conceived the project. HEB, LC, OAK, RXS, KRB, MLU, EJ, JLR, JL, and KEF designed the experiments. HEB, LC, 
OAK, RXS, and KRB managed data. HEB, OAK, and RR wrote the original draft. $\mathrm{HEB}, \mathrm{OAK}, \mathrm{KEF}$, and RR reviewed and edited the manuscript. HEB and RR acquired funding. AMMM, RD, and RR provided resources. RR supervised the project.

\section{Acknowledgments}

This research was supported by a Canadian Institutes of Health Research (CIHR) Foundation grant, a BioCanRX Catalyst grant, a Cancer Research Society operating grant, and a grant from the Princess Margaret Foundation. This study used resources provided by the Canadian Ovarian Cancer Research Consortium's bio- bank funded by the Terry Fox Research Institute (grant 2012-46). AMMM is a researcher at CHUM, which receives support from the Fonds de Recherche du Québec (FRQS). We thank UHN animal and STARR facilities for assistance with Xenogen and Napoleon Law at STARR for help with IHC.

Address correspondence to: Robert Rottapel or Helen E. Burston, Princess Margaret Cancer Centre, 101 College Street TMDT 12-701, Toronto, Ontario M5G 1L7, Canada. Phone: 416.581.7852; Email: rottapel@gmail.com (RR). Phone: (416)-567-0807, hburston@cmmt.ubc.ca (HEB).
1. Dong A, et al. Genomic/epigenomic alterations in ovarian carcinoma: translational insight into clinical practice. J Cancer. 2016;7(11):1441-1451.

2. Lengyel E. Ovarian cancer development and metastasis. Am J Pathol. 2010;177(3):1053-1064.

3. Bast RC, et al. The biology of ovarian cancer: new opportunities for translation. Nat Rev Cancer. 2009;9(6):415-428.

4. Yap TA, et al. Beyond chemotherapy: targeted therapies in ovarian cancer. Nat Rev Cancer. 2009;9(3):167-181.

5. O'Hayre M, et al. The emerging mutational landscape of G proteins and G-protein-coupled receptors in cancer. Nat Rev Cancer. 2013;13(6):412-424.

6. Liu Y, et al. G protein-coupled receptors as promising cancer targets. Cancer Lett. 2016;376(2):226-239.

7. Bathgate RAD, et al. Relaxin family peptides and their receptors. Physiol Rev. 2013;93(1):405-480.

8. Marshall SA, et al. The role of relaxin in normal and abnormal uterine function during the menstrual cycle and early pregnancy. Reprod Sci. 2017;24(3):342-354.

9. Dessauer CW, Nguyen BT. Relaxin stimulates multiple signaling pathways: activation of cAMP, PI3K, and PKC $\zeta$ in THP-1 cells. Ann N Y Acad Sci. 2005;1041:272-279.

10. Thanasupawat T, et al. Emerging roles for the relaxin/RXFP1 system in cancer therapy. Mol Cell Endocrinol. 2019;487:85-93.

11. Medrano $\mathrm{M}$, et al. Interrogation of functional cell-surface markers identifies CD151 dependency in high-grade serous ovarian cancer. Cell Rep. 2017;18(10):2343-2358.

12. Marcotte R, et al. Essential gene profiles in breast, pancreatic, and ovarian cancer cells. Cancer Discov. 2012;2(2):172-189.

13. Domcke S, et al. Evaluating cell lines as tumour models by comparison of genomic profiles. Nat Commun. 2013;4:2126.

14. Beaufort CM, et al. Ovarian cancer cell line panel (OCCP): clinical importance of in vitro morphological subtypes. PLoS One. 2014;9(9):e103988.

15. Halls ML, et al. Relaxin family peptide receptors--former orphans reunite with their parent ligands to activate multiple signalling pathways. Br J Pharmacol. 2007;150(6):677-691.

16. Rodgers LH, et al. Loss of PAX8 in high-grade serous ovarian cancer reduces cell survival despite unique modes of action in the fallopian tube and ovarian surface epithelium. Oncotarget. 2016;7(22):32785-32795.

17. Pontén F, et al. The Human Protein Atlas--a tool for pathology. J Pathol. 2008;216(4):387-393.

18. Unemori EN, et al. Relaxin induces vascular endothelial growth factor expression and angiogenesis selectively at wound sites. Wound Repair Regen. 2000;8(5):361-370.

19. Ahmad N, et al. Relaxin induces matrix-metalloproteinases- 9 and -13 via RXFP1: induction of MMP-9 involves the PI3K, ERK, Akt and PKC- $\zeta$ pathways. Mol Cell Endocrinol. 2012;363(1-2):46-61.

20. Meyers RM, et al. Computational correction of copy number effect improves specificity of CRISPR-Cas 9 essentiality screens in cancer cells. Nat Genet. 2017;49(12):1779-1784.

21. Dempster JM, et al. Extracting biological insights from the project achilles genome-scale CRISPR screens in cancer cell lines [preprint]. https://doi.org/10.1101/720243. Posted on bioRxiv July 31, 2019.

22. Ghandi M, et al. Next-generation characterization of the Cancer Cell Line Encyclopedia. Nature. 2019;569(7757):503-508.

23. Cerami E, et al. The cBio cancer genomics portal: an open platform for exploring multidimensional cancer genomics data. Cancer Discov. 2012;2(5):401-404.

24. Gao J, et al. Integrative analysis of complex cancer genomics and clinical profiles using the cBioPortal. Sci Signal. 2013;6(269):pl1.

25 . Bell D, et al. Integrated genomic analyses of ovarian carcinoma. Nature. 2011;474(7353):609-615

26. Halls ML, et al. International Union of Basic and Clinical Pharmacology. XCV. Recent advances in the understanding of the pharmacology and biological roles of relaxin family peptide receptors 1-4, the receptors for relaxin family peptides. Pharmacol Rev. 2015;67(2):389-440.

27. Bruell S, et al. Chimeric RXFP1 and RXFP2 receptors highlight the similar mechanism of activation utilizing their $\mathrm{N}$-terminal low-density lipoprotein class A modules. Front Endocrinol. (Lausanne). 2013;4:171.

28. Sudo S, et al. H3 relaxin is a specific ligand for LGR7 and activates the receptor by interacting with both the ectodomain and the exoloop 2 . J Biol Chem. 2003;278(10):7855-7862.

29. Radestock Y, et al. Relaxin enhances S100A4 and promotes growth of human thyroid carcinoma cell xenografts. Mol Cancer Res. 2010;8(4):494-506

30. Neschadim A, et al. Relaxin receptor antagonist AT-001 synergizes with docetaxel in androgenindependent prostate xenografts. Endocr Relat Cancer. 2014;21(3):459-471.
31. Lee Y, et al. A candidate precursor to serous carcinoma that originates in the distal fallopian tube. JPathol. 2007;211(1):26-35.

32. Tang XM, Chegini N. Human fallopian tube as an extraovarian source of relaxin: messenger ribonucleic acid expression and cellular localization of immunoreactive protein and 125I-relaxin binding sites. Biol Reprod. 1995;52(6):1343-1349.

33. Rouillard AD, et al. The harmonizome: a collection of processed datasets gathered to serve and mine knowledge about genes and proteins. Database (Oxford). 2016;2016:baw100.

34. Ahmed N, Stenvers KL. Getting to know ovarian cancer ascites: opportunities for targeted therapy-based translational research. Front Oncol. 2013;3:256.

35. Kim S, et al. Ascites modulates cancer cell behavior, contributing to tumor heterogeneity in ovarian cancer. Cancer Sci. 2016;107(9):1173-1178.

36. Kolomeyevskaya N, et al. Cytokine profiling of ascites at primary surgery identifies an interaction of tumor necrosis factor- $\alpha$ and interleukin- 6 in predicting reduced progression-free survival in epithelial ovarian cancer. Gynecol Oncol. 2015;138(2):352-357.

37. Macciò A, Madeddu C. Inflammation and ovarian cancer. Cytokine. 2012;58(2):133-147.

38. Browning L, et al. IL-6 and ovarian cancer: inflammatory cytokines in promotion of metastasis. Cancer Manag Res. 2018;10:6685-6693.

39. Horton JS, et al. Relaxin modulates proinflammatory cytokine secretion from human decidual macrophages. Biol Reprod. 2011;85(4):788-797.

40. Horton JS, et al. Relaxin augments the inflammatory IL6 response in the choriodecidua. Placenta. 2012;33(5):399-407.

41. Tempfer C, et al. Serum evaluation of interleukin 6 in ovarian cancer patients. Gynecol Oncol. 1997;66(1):27-30.

42. Jones VS, et al. Cytokines in cancer drug resistance: Cues to new therapeutic strategies. Biochim Biophys Acta. 2016;1865(2):255-265.

43. Zhao Y, et al. Cytotoxicity enhancement in MDA-MB-231 cells by the combination treatment of tetrahydropalmatine and berberine derived from Corydalis yanhusuo W. T. Wang. J Intercult Ethnopharmacol.2014;3(2):68-72.

44. Matei D, et al. Autocrine activation of PDGFRalpha promotes the progression of ovarian cancer. Oncogene. 2006;25(14):2060-2069.

45. Xu Y. Lysophospholipid signaling in the epithelial ovarian cancer tumor microenvironment. 
Cancers (Basel). 2018;10(7):227.

46. Clayton NS, et al. Fibroblast growth factormediated crosstalk in cancer etiology and treatment. Dev Dyn. 2017;246(7):493-501.

47. Gera $\mathrm{S}$, et al. Follicle-stimulating hormone is an autocrine regulator of the ovarian cancer metastatic niche through notch signaling. J Endocr Soc. 2019;3(2):340-357.

48. Groeneweg JW, et al. Notch signaling in serous ovarian cancer. JOvarian Res. 2014;7:95.

49. Brzozowa-Zasada M, et al. Notch and its oncogenic activity in human malignancies. Eur Surg. 2017;49(5):199-209.

50. Wang Y, et al. Interleukin-6 signaling regulates anchorage-independent growth, proliferation, adhesion and invasion in human ovarian cancer cells. Cytokine. 2012;59(2):228-236.
51. Coward J, et al. Interleukin- 6 as a therapeutic target in human ovarian cancer. Clin Cancer Res. 2011;17(18):6083-6096.

52. Saini U, et al. STAT3/PIAS3 levels serve as "early signature" genes in the development of highgrade serous carcinoma from the fallopian tube. Cancer Res. 2018;78(7):1739-1750.

53. Feng $S$, et al. Developmental expression and gene regulation of insulin-like 3 receptor RXFP2 in mouse male reproductive organs. Biol Reprod. 2007;77(4):671-680.

54. Jo A, et al. The versatile functions of Sox 9 in development, stem cells, and human diseases. Genes Dis. 2014;1(2):149-161.

55. Matheu A, et al. Oncogenicity of the developmental transcription factor Sox9. Cancer Res. 2012;72(5):1301-1315.
56. Siddik ZH. Cisplatin: mode of cytotoxic action and molecular basis of resistance. Oncogene. 2003;22(47):7265-7279.

57. Pogge von Strandmann E, et al. Tumor-host cell interactions in ovarian cancer: pathways to therapy failure. Trends Cancer. 2017;3(2):137-148.

58. Wang Y, et al. [Chemotherapy resistance induced by interleukin- 6 in ovarian cancer cells and its signal transduction pathways]. Zhonghua Fu Chan Ke Za Zhi. 2010;45(9):691-698.

59. Guo $X$, et al. Serum relaxin as a diagnostic and prognostic marker in patients with epithelial ovarian cancer. Cancer Biomark. 2017;21(1):81-87.

60. Lounis H, et al. Primary cultures of normal and tumoral human ovarian epithelium: a powerful tool for basic molecular studies. Exp Cell Res. 1994;215(2):303-309. 